Check for updates

Cite this: RSC Adv., 2017, 7, 27578

\title{
Biomedical films of graphene nanoribbons and nanoflakes with natural polymers
}

\author{
Magda Silva, abc Sofia G. Caridade, ${ }^{\text {ab }}$ Ana C. Vale, (D) bunice Cunha, ${ }^{\mathrm{c}}$ Maria P. Sousa, ${ }^{\text {ab }}$ \\ João F. Mano, ${ }^{\text {ab }}$ Maria C. Paiva*c and Natália M. Alves (iD *ab
}

Graphene and its derivatives are promising as reinforcement for polymer nanocomposites. Additionally to their inherent outstanding mechanical properties, these nanoparticles may be functionalized to enhance their compatibility with the polymeric matrix and provide specific chemical and physical properties. In this work, new freestanding films (FS) based on chitosan ( $\mathrm{CHI}$ ), alginate (ALG) and functionalized graphene were developed using the layer-by-layer assembly. Suspensions of functionalized graphene nanoflakes (f-GF) and nanoribbons ( $f$-GNR) were prepared from expanded graphite (EG) and multiwalled carbon nanotubes (MWNTs), respectively. The graphene nanoflakes and MWNTs were covalently functionalized using a 1,3-dipolar cycloaddition reaction that allowed the nanoparticles exfoliation. fGNR and f-GF suspensions were characterized to demonstrate that graphene nanoflakes and MWNTs were successfully functionalized and exfoliated. Then, the layer-by-layer deposition of CHI, ALG and both types of functionalized graphene was investigated and FS films were produced. The morphology, thermal and mechanical characteristics of the produced FS films were assessed. Their degradation and swelling profiles as well as their biological behavior were evaluated. The incorporation of $f$-GF resulted in smoother films while the incorporation of $f-G N R$ resulted in rougher films. When compared with the $\mathrm{CHI} / \mathrm{ALG}$ bi-component films. Both graphene containing films remained hydrophobic. The graphene incorporation in the multilayered FS was estimated to be $1.7 \mathrm{wt} \%$ for $\mathrm{f}-\mathrm{GF}$ and $2.5 \mathrm{wt} \%$ for $\mathrm{f}$-GNR. The presence of functionalized graphene did not affect the thermal stability of the films, it increased the storage modulus and the dynamic mechanical response at $1 \mathrm{~Hz}$ and $37{ }^{\circ} \mathrm{C}$, and decreased the electrical resistivity. The biological assays revealed cytocompatibility towards $L 929$ cells when both $\mathrm{f}$-GF and $f$ GNR were incorporated in the CHI/ALG matrix. In conclusion, these new $f$-GF and $f$-GNR reinforced FS films present great potential for use in biomedical applications such as films for wound healing or cardiac and bone engineering.

rsc.li/rsc-advances

\section{Introduction}

Graphene is a planar sheet of $\mathrm{sp}^{2}$ hybridized carbon atoms densely arranged into a $2 \mathrm{D}$ honeycomb lattice. ${ }^{1-3}$ It is a zero-gap semiconductor ${ }^{4}$ and the base structure of carbon allotropes such as nanotubes and graphite. ${ }^{5}$ It presents a high elastic modulus $(\approx 1 \mathrm{TPa})$ and a tensile strength of $130 \pm 10 \mathrm{GPa} \cdot{ }^{6,7} \mathrm{It}$ also has the ability to sustain extremely high electric current densities (a million times higher than copper) and presents a high thermal conductivity, above $3000 \mathrm{~W} \mathrm{~m}^{-1} \mathrm{~K}^{-1}, 7$ and a large theoretical specific area of $2630 \mathrm{~m}^{2} \mathrm{~g}^{-1} \cdot{ }^{8}$ Besides, most studies indicate that graphene and its derivatives are

${ }^{a} 3 B$ 's Research Group, Biomaterials, Biodegradables and Biomimetics, University of Minho, Headquarters of the European Institute of Excellence on Tissue Engineering and Regenerative Medicine, AvePark-Parque de Ciência e Tecnologia, 4805-017 Barco, Taipas, Guimarães, Portugal.E-mail:nalves@dep.uminho.pt ${ }^{b} I C V S / 3 B$ 's, Associate PT Government Laboratory, Braga/Guimarães, Portugal 'Institute for Polymers and Composites/I3N, Department of Polymer Engineering, University of Minho, 4800-058 Guimarães, Portugal. E-mail: mcpaiva@dep.uminho.pt biocompatible and have low toxicity, although no consensus has been reached yet. ${ }^{9}$ Due to these outstanding characteristics, graphene and its derivatives have been widely investigated in a range of applications including in the biomedical field, such as biosensing/bioimaging, ${ }^{3}$ antibacterial materials, ${ }^{10}$ and drug/ gene delivery. ${ }^{9-11}$ Graphene has also been used as a filler in medical implants, hydrogels and tissue engineering scaffolds. ${ }^{8}$ However, graphene is hydrophobic and presents poor dispersion in organic and aqueous solvents containing proteins, salts or other ions. The functionalization of graphene appears as a suitable strategy to allow the production of stable suspensions $^{\mathbf{3 , 8 , 1 1 , 1 2}}$ and to enhance the compatibility between graphene and a polymeric matrix, ${ }^{6,13}$ which results in the production of composites with exceptional structural properties at low reinforcement loads. ${ }^{\mathbf{1 4}}$ The functionalization of graphene may be achieved via covalent and non-covalent modifications. The former leads to the formation of strong and stable interfaces with polymeric matrices..$^{3,15}$ 
Functionalized graphene nanoribbons (f-GNR) and functionalized graphene nanoflakes (f-GF) can be achieved by exfoliation, directly from functionalized multi-walled carbon nanotubes (f-MWNTs) and expanded graphite (f-EG), respectively. ${ }^{\mathbf{1 5}, 16}$ The covalent functionalization based on the 1,3dipolar cycloaddition reaction of azomethine ylides (DCA) ${ }^{15,17}$ demonstrated to induce the unzipping of carbon nanotubes, ${ }^{18}$ and showed a similar effect on graphene nanoflakes. ${ }^{16}$ DCA is a simple one-pot reaction which allows the homogenous functionalization at large scale. Comparing to the covalent oxidation of graphene that produces a significant amount of structural defects, ${ }^{19,20}$ DCA is not oxidative and does not affect the graphene structure, thus the electronic properties of graphene are preserved, being useful for further applications. ${ }^{18}$

Natural polymers have been extensively used in biomedical applications due to their inherent biocompatibility and biodegradability. ${ }^{21}$ Chitosan (CHI) is a natural cationic polymer obtained through deacetylation of chitin by hydrolysis. ${ }^{22-25}$ As a polyelectrolyte, $\mathrm{CHI}$ can notably be employed for the preparation of multilayered films, using the layer-by-layer (LbL) deposition technique. ${ }^{23} \mathrm{CHI}$ possesses a number of interesting properties including biocompatibility, non-toxicity and biodegradability, a remarkable affinity to proteins, antibacterial, fungi-static and anti-tumoral properties. ${ }^{26}$ Alginate (ALG) is a natural anionic polymer which can be extracted from brown algae or provided by bacterial biosynthesis. ${ }^{25,27,28}$ Due to its biocompatibility, low-toxicity, biodegradation, gel-forming ability and low cost, ALG has found numerous applications in the biomedical field, such as wound healing, drug delivery and scaffolds for tissue engineering. ${ }^{27,28}$

LbL is a simple, robust, cost-effective, flexible, and versatile bottom up strategy to modify surfaces and fabricate functional and thin multilayered films with tailored thickness, composition, structure, properties and functions. It consists on the sequential adsorption of polyelectrolytes with opposite charge onto a substrate surface, ${ }^{29,30}$ via strong electrostatic forces and also non-electrostatic forces such as hydrophobic interactions. ${ }^{31}$ Different multilayered devices may result with distinct shapes, sizes and functionality such as freestanding (FS) films. ${ }^{30,32} \mathrm{LbL}$ structures have been employed for a variety of biomedical applications, such as in the coating of implantable devices, biosensors, in drug/gene delivery, in tissue engineering and regenerative medicine. ${ }^{30}$

The major disadvantages of natural polymers are their poor mechanical and electrical properties. ${ }^{33}$ To overcome these drawbacks, the reinforcement of natural polymers with graphene and its derivatives has been reported, in order to synergistically combine the strengths of the components and optimize the primary properties. ${ }^{34}$ The resultant graphene based-composites have shown significant improvement in the mechanical properties as well as in the electrical conductivity. ${ }^{35}$ Other advantages have been reported such as the increase of cellular attachment and growth at biomaterials surface and the production of more efficient biosensors. ${ }^{9}$

A considerable number of studies regarding LbL assembly with graphene, graphene oxide (GO) and reduced graphene oxide (rGO) have been published. However, only a reduced number of publications reported the production of composite materials with graphene and natural polymers, using LbL assembly. Barsan et $a{ }^{36}{ }^{36}$ reported the construction of a high sensitivity biosensor through LbL technique. Multilayer films based on glucose oxidase and nitrogen-doped graphene both dispersed in the positively-charged $\mathrm{CHI}$ and a negatively charged polymer poly(styrene sulfonate) were assembled by alternately immersing a gold electrode substrate in the two aforementioned solutions. ${ }^{36}$ Tang and co-workers produced multilayer films of regenerated cellulose and GO by LbL assembly with enhanced mechanical properties and electrical conductivity, which make them promising for advanced biochemical and electrochemical devices. ${ }^{37}$ We have also reported the production of FS films, based on CHI, ALG and GO (from oxidized MWNTs and EG) using LbL assembly, in which the produced FS films presented interesting properties for biomedical applications. ${ }^{38}$

According to the above observations, the present work proposes for the first time the production of FS multilayered films based on functionalized graphene, CHI and ALG using LbL assembly, in which functionalized graphene (f-GF or f-GNR) is used as nanofiller to reinforce the intrinsic properties of the polymeric matrix. The produced FS films could be potentially used in different biomedical applications, namely for tissue engineering applications or wound healing. Initially, the produced functionalized graphene was characterized by ultraviolet-visible spectroscopy (UV-Vis), Raman spectroscopy and thermogravimetric analysis (TGA). Before the production of multilayered films, quartz crystal microbalance with dissipation (QCM-D) experiments were performed to investigate the best conditions for the buildup of the films. After that, FS films were produced and characterized by scanning electron microscopy (SEM), atomic force microscopy (AFM) and TGA. The mechanical performance, wettability, water uptake, degradation and biological behavior of the obtained FS films were also evaluated.

\section{Experimental section}

\subsection{Materials}

MWNTs (purity approx. 90\%; average length $=1.5 \mu \mathrm{m}$ ) were supplied by Nanocyl ${ }^{\mathrm{TM}}$, Belgium (NC7000) and exfoliated graphite (EG) (purity approx. 99.5\%; average length $=9.9 \mu \mathrm{m}$ ) was obtained from Nacional de Grafite Ltda., Brazil (Micrograf HC11). The surface of MWNTs and EG was covalently functionalized through the DCA reaction using the amino acid $N$ benzyloxycarbonylglycine (Z-Gly-OH, 99\%, from Aldrich), and paraformaldehyde (reagent grade) from Sigma-Aldrich. The reagents were mixed with MWNTs or EG using a small amount of diethyl ether (stabilized with $\sim 6$ ppm de BHT, from Panreac). The functionalization was carried out according to the procedure described for MWCNT. ${ }^{15}$ The washing of the functionalized products was carried out using absolute ethanol from Fisher Scientific UK, commercial hexane from JMS and acetone from Pronalab.

Both functionalized multi-walled nanotubes (f-MWNTs) and functionalized expanded graphite (f-EG) suspensions were prepared in ethanol absolute (from Fisher Scientific UK) for 
further exfoliation and posterior addition of distilled water (DW).

Regarding the production of the FS films, CHI (medium molecular weight) was purchased from Sigma-Aldrich (Germany), with a degree of $N$-deacetylation (DD) ranging from $75-$ $85 \%$ and a viscosity of $200-800 \mathrm{cps}$. Prior to be used, CHI was purified by a recrystallization process. Sodium alginate from brown algae (ALG) was purchased by Sigma-Aldrich, with a viscosity of 5 to $40 \mathrm{cps}$.

\subsection{Production of functionalized graphene suspensions}

Functionalized graphene nanoribbons (f-GNR) and functionalized graphene nanoflakes (f-GF) suspensions were obtained directly from f-MWNTs and f-EG, through exfoliation, as described by Paiva et al. ${ }^{15}$ Both f-MWNTs and f-EG were attained through surface functionalization using a 1,3 dipolar cycloaddition of azomethine ylides (DCA) reaction. ${ }^{15}$ It involved the mixture of $5 \mathrm{~g}$ of $\mathrm{N}$-benzyloxy-carbonylglycine with $50 \mathrm{~mL}$ of diethyl ether. This suspension was left under magnetic stirring, at room temperature, during $5 \mathrm{~min}$. After that, $3.5 \mathrm{~g}$ of paraformaldehyde was added. Once again, the suspension was kept under magnetic stirring, at room temperature during $5 \mathrm{~min}$. Afterwards, $5 \mathrm{~g}$ of EG or MWNTs and $50 \mathrm{~mL}$ of ethyl ether were added to the previous mixture, forming a slurry that was stirred and heated to $30-35{ }^{\circ} \mathrm{C}$, to evaporate ethyl ether, leaving an homogeneous solid mixture. Then, the temperature was increased to $250{ }^{\circ} \mathrm{C}$ and maintained for $3 \mathrm{~h}$. During this stage paraformaldehyde decomposes into formaldehyde, that reacts with the melted Z-Gly-OH forming the corresponding 1,3dipole. The dipole reacts with the surface of MWNTs or EG through cycloaddition, to originate a cyclic amine, or pyrrolidine. The resultant products were cooled, washed with ethanol absolute, hexane, acetone and ethanol again. The products were dried at $80{ }^{\circ} \mathrm{C}$ for 5 hours. After functionalization of MWNTs and $\mathrm{EF}$, two suspensions of graphene were produced in ethanol absolute, namely with f-GNR and f-GF, following the procedure described before for f-GNR. ${ }^{51}$ For that purpose, $0.5 \mathrm{~g}$ of $\mathrm{f}$ MWNTs or f-EG were suspended in $500 \mathrm{~mL}$ of ethanol absolute. Their unzipping/exfoliation was carried out by subjecting the suspensions to ultrasounds during 4 hours, in an ultrasound bath Sonorex Plus RK 102H from Bandelin. After that, the suspensions were vacuum filtered using a Vacuum pump (DS102, Varian) and then, by action of gravity using a PTFE membrane filter (Whatman, UK). The resultant ethanol suspensions of f-GNR and f-GF were then adjusted to obtain suspensions with a concentration of $0.25 \mathrm{mg} \mathrm{mL}^{-1}$ in a mixture of $25 \%$ ethanol and $75 \%$ DW. These percentages were established after an optimization process developed in another study. ${ }^{39}$

\subsection{Structural characterization of $\mathbf{f}-\mathrm{GF}$ and f-GNR}

Raman spectroscopy analysis was performed on a LabRAM HR Evolution Raman spectrometer (Horiba Scientific, Japan) with a laser excitation wavelength of $532 \mathrm{~nm}$ and the results were analyzed using the LabSpec6 software. The pristine MWNTs and EG as well as f-MWNTs, f-EG and functionalized few-layer graphene formed in suspension (f-GNR and f-GF) were sprayed on glass slides positioned on a heating plate for fast solvent evaporation and deposition of the graphene products to be analyzed. UV-Vis spectroscopy analysis of the ethanol suspensions was also performed for additional evidence for the formation of graphene and to determine the concentration of the few-layer functionalized graphene in suspension, using a UV-2401 PC spectrometer (Shimadzu, Japan) and quartz cells with $1 \mathrm{~cm}$ path length. The thermal stability of $\mathrm{f}-\mathrm{EG}$ and $\mathrm{f}$ MWNTs was evaluated by thermogravimetric analysis (TGA), performed on a TA Q500 equipment (TA Instruments, USA). The measurements were carried out under a nitrogen flow of $50 \mathrm{~mL}$ $\min ^{-1}$, within a temperature range from $40{ }^{\circ} \mathrm{C}$ to $800^{\circ} \mathrm{C}$, and at a scanning rate of $10^{\circ} \mathrm{C} \mathrm{min}^{-1}$.

\subsection{Real-time monitoring of multilayered films}

The buildup of the multilayered films was monitored in real time using a Quartz Crystal Microbalance with dissipation (QCM-D). CHI (polycation), (f-GF or f-GNR)-ALG (polyanions) were combined to form 6 bilayers.

CHI was dissolved overnight in a mixture of $75 \%$ DW/ $25 \%$ ethanol and $1 \%(\mathrm{v} / \mathrm{v})$ acetic acid $\left(c=0.5 \mathrm{mg} \mathrm{mL}^{-1}\right) \cdot{ }^{45}$ Regarding (f-GF or f-GNR)-ALG solutions, an appropriate amount of ALG was dissolved in each graphene suspension (previously prepared with $c=0.25 \mathrm{mg} \mathrm{mL}{ }^{-1}$ ) in order to fulfill a concentration of $0.5 \mathrm{mg} \mathrm{mL}^{-1}$. A washing solution (mixture of $75 \%$ $\mathrm{DW} / 25 \%$ ethanol) was also prepared. All solutions were adjusted to $\mathrm{pH}$ 5.5.

Six bilayers of both (CHI/f-GNR-ALG) ${ }_{6}$ and (CHI/f-GF-ALG) 6 were assembled, the adsorption time was $10 \mathrm{~min}$ and $5 \mathrm{~min}$ for the polyelectrolytes and the washing solution, respectively. The washing solution was used between each polyelectrolyte deposition.

All QCM-D experiments were performed at $25^{\circ} \mathrm{C}$ with a $50 \mu \mathrm{L}$ $\min ^{-1}$ flow rate in a QCM-D E4 from Q-Sense Instruments, Sweden. The QCM-D experimental data of the obtained viscoelastic films were modelled with a QTools software from Qsense $\mathrm{AB}$, according to a Voigt based model and using 3 overtones of frequency and dissipation $\left(3^{\text {rd }}, 5^{\text {th }}\right.$ and $\left.7^{\text {th }}\right)$. The solvent viscosity selected was $1 \mathrm{mPa} s$ and a film density of $1 \mathrm{~g} \mathrm{~cm}^{-3}$. The solvent density was varied between values from 1000 to $1030 \mathrm{~kg} \mathrm{~m}^{-3}$ in order to minimize the total error $\left(\chi^{2}\right) \cdot{ }^{40-42}$

\subsection{Production of the FS multilayered films}

Three types of FS films composed of 200 bilayers were produced using a homemade dipping robot. The films were produced on polypropylene (PP) supports and were named as (CHI/f-GF$\mathrm{ALG})_{200}$, (CHI/f-GNR-ALG) $)_{200}$ and (CHI/ALG) ${ }_{200}$. The (CHI/ ALG $)_{200}$ FS films were use as control. Before the buildup, the PP supports were thoroughly washed with ethanol and DW and dried with compressed air. CHI, f-GF and f-GF-ALG solutions were prepared as described in Section 2.4 but with a concentration of $2 \mathrm{mg} \mathrm{mL}^{-1}$. $^{45}$ Regarding f-GF and f-GNR-ALG solutions, an appropriate amount of ALG was dissolved in each graphene suspension in order to fulfill a concentration of $2 \mathrm{mg}$ 
$\mathrm{mL}^{-1}$. A washing solution (mixture of $75 \% \mathrm{DW} / 25 \%$ ethanol) was also prepared. All solutions were adjusted to $\mathrm{pH} 5.5$.

PP substrates were immersed in the polyelectrolyte solutions (CHI and f-GF or f-GNR-ALG) during $6 \mathrm{~min}$, intercalated with rinsing steps of $4 \mathrm{~min}$.

\subsection{Thermogravimetric analysis}

Thermogravimetric analysis was performed on a TA Q500 equipment (TA Instruments, USA). Approximately $4 \mathrm{mg}$ of each FS film was cut for the experiments. The process undergone using a nitrogen atmosphere with a temperature range between $40{ }^{\circ} \mathrm{C}$ and $800{ }^{\circ} \mathrm{C}$ and at a scanning rate of $10^{\circ} \mathrm{C} \mathrm{min}^{-1}$.

\subsection{Scanning electron microscopy of the FS films}

The morphologies of the upper side (facing outward) of the FS films were characterized, using a JSM-6010LV SEM (JEOL, Japan), operating at $15 \mathrm{kV}$ accelerating voltage. The samples were previously sputtered with a gold layer, using a sputter coater 108A (Cressington, UK). For the cross-section observations, the FS were dipped in liquid nitrogen until free fracture.

\subsection{Atomic force microscopy imaging}

In order to analyze their topography and roughness, dried FS films were imaged using a Dimension Icon AFM equipment (Bruker, France) with an air cantilever (SNL-10D) (Bruker, France) with a spring constant of $0.06 \mathrm{~N} \mathrm{~m}^{-1}$, operating in a ScanAsyst mode. The topography of the FS films was analyzed with $512 \times 512$ pixels $^{2}$ at line rates of $1 \mathrm{~Hz}$. For surface roughness analysis, AFM images with $5 \times 5 \mu^{2}$ were obtained, followed by root mean squared roughness $\left(R_{\mathrm{RMS}}\right)$ and average height value $\left(H_{\mathrm{AV}}\right)$ calculation. At least three measurements were done for each type of film. The analysis of the obtained images was performed using NanoScope Analysis software.

\subsection{Contact angle measurements}

In order to evaluate the wettability of the film surfaces, water contact angle measurements were performed using a goniometer equipment model OCA15plus from DataPhysics Instruments (Filderstadt, Germany). The sessile drop method was applied, in which a $5 \mu \mathrm{L}$ drop of pure water was deposited on the sample surface by a syringe. As the water drop contacted the surface, images were acquired and analyzed using the SCA 20 software. At least three measurements of each condition were performed on each film surface.

\subsection{Swelling ability studies}

The swelling ability of the produced FS films was measured by soaking dry films (previously weighed), in phosphate buffer saline solution (PBS; Sigma, USA) at $37{ }^{\circ} \mathrm{C}$ during $5 \mathrm{~min}, 15 \mathrm{~min}$, $30 \mathrm{~min}, 1 \mathrm{~h}, 3 \mathrm{~h}, 5 \mathrm{~h}, 7 \mathrm{~h}, 24 \mathrm{~h}$ and $48 \mathrm{~h}$. After each soaking period, the FS were taken from PBS solution, the excess was removed with a filter paper and the FS were immediately weighted. The water absorption percentage was calculated according to eqn (1): ${ }^{43}$

$$
\text { Water uptake }(\%)=\frac{W_{\mathrm{w}}-W_{\mathrm{d}}}{W_{\mathrm{d}}} \times 100,
$$

where $W_{\mathrm{w}}$ is the weight of the FS in the swollen state at a determined time point and $W_{\mathrm{d}}$ is the dry weight of the FS.

\subsection{Weight loss}

The weight loss of all produced FS films caused by nonenzymatic hydrolysis and enzyme-catalyzed hydrolysis, which are two common degradation processes of natural polymers, was evaluated by immersing previously dry weighed samples in two different solutions: (i) a solution of PBS and (ii) a solution of PBS with lysozyme from chicken egg white (Sigma-Aldrich) at a concentration of $13 \mathrm{mg} \mathrm{mL}{ }^{-1}$. The samples were stored in an incubator for 7, 14, 21 and 28 days, at $37^{\circ} \mathrm{C}$. All solutions were changed every two days. At each time point, the samples were removed from the solution, washed with distilled water and dried and, subsequently weighed. The weight loss was calculated using eqn (2): ${ }^{43}$

$$
\text { Weight loss }(\%)=\frac{\left(W_{\mathrm{i}}-W_{\mathrm{f}}\right)}{W_{\mathrm{i}}} \times 100,
$$

where $W_{\mathrm{i}}$ is the initial dry weight of the sample before immersion and $W_{\mathrm{f}}$ is the final mass of the dried sample at a given time point. Each experiment was repeated three times and the average value was taken as the weight loss.

\subsection{Dynamical mechanical analysis}

In order to evaluate the mechanical/viscoelastic properties of the FS, DMA tests were performed using a Tritec 2000B equipment (Triton Technology, UK), equipped with the tensile mode. Samples were cut with a width of $5 \mathrm{~mm}$ and a length of $20 \mathrm{~mm}$, approximately. The thickness of each sample was determined in three different points, using a micrometer (Mitutoyo, Japan). Previously to the DMA tests, the samples were soaked overnight, in a PBS solution, to reach the swelling equilibrium. FS films were clamped in the DMA apparatus with a grip distance of 10 $\mathrm{mm}$ and with total immersion of the sample in a Teflon ${ }^{\circledR}$ reservoir containing PBS. The measurements were carried out at $37{ }^{\circ} \mathrm{C}$. After equilibrating at $37{ }^{\circ} \mathrm{C}$, the DMA spectra were obtained using a stress mode cycle of increasing frequency from $0.1 \mathrm{~Hz}$ to $1 \mathrm{~Hz}$, in order to simulate the physiological loading frequencies. ${ }^{\mathbf{4 4}}$ A static pre-load of $1 \mathrm{~N}$ was applied during the tests to keep the sample tight. At least three specimens were tested for each condition.

\subsection{Electrical resistivity measurements}

The electrical measurements were performed following ASTM D257, using a picoammeter 6487 (Keithley, USA). By varying the voltage applied from -5.0 to $5.0 \mathrm{~V}$, the current intensity was measured using direct current measurements. All samples were rectangular with an area of $1.68 \times 10^{-4} \mathrm{~m}^{2}$ and their thickness was measured before the test, using a micrometer (Mitutoyo, Japan). The results presented are the average of 100 measurements performed, for each applied voltage. 


\subsection{Biological assays}

2.14.1. Cell culturing and seeding. L929 mouse fibroblasts line (L929, European Collection of Cell Cultures) were used in order to test the in vitro biocompatibility of the (CHI/f-GF$\mathrm{ALG})_{200}$, (CHI/f-GNR-ALG) $)_{200}$ and (CHI/ALG) $)_{200}$ for 1, 3 and 7 days. Cells were cultured in complete Dulbecco's modified minimum essential medium (DMEM) medium supplemented with $3.7 \mathrm{~g} \mathrm{~L}^{-1}$ sodium bicarbonate, 10\% FBS and 1\% penicillinstreptomycin ( $\mathrm{pH} 7.4)$, in $150 \mathrm{~cm}^{2}$ tissue culture flasks and incubated at $37{ }^{\circ} \mathrm{C}$ in a humidified air atmosphere of $5 \% \mathrm{CO}_{2}$. The medium was replaced, every $2-3$ days.

Before cell seeding, samples with $10 \times 10 \mathrm{~mm}^{2}$ were sterilized by immersion in $70 \%(\mathrm{v} / \mathrm{v})$ ethanol for $2 \mathrm{~h}$ and by exposition to UV light during $1 \mathrm{~h}$. After, all FS films were washed twice with PBS and were immersed in cell culture medium for complete swelling. The cells were seeded at a density of 50000 cells per sample and nourished with DMEM. The cultures were incubated at $37{ }^{\circ} \mathrm{C}$ in a humidified air atmosphere of $5 \% \mathrm{CO}_{2}$. The same seeding procedure was used on TCPS in order to use them as positive control.

2.14.2. DAPI-phalloidin staining. The morphology and viability of the L929 cells was evaluated using fluorescence staining with phalloidin tetramethylrhodamine and $4^{\prime}$,6-diamidino-2-phenylindole (DAPI). At each time point DAPI (1:1000 in PBS, $\mathrm{pH}=7.4$, Sigma Aldrich, USA) and phalloidin (1 : 150 in PBS, $\mathrm{pH}=7.4$, Sigma Aldrich, USA) were used. Before staining, culture medium was removed and the samples were washed with sterile PBS and fixed by using formalin (10\%, ThermoFisher) for $30 \mathrm{~min}$. After that, formalin was removed and the samples were washed with PBS. Following this, phalloidin was added, protected from the light and kept at room temperature during $45 \mathrm{~min}$. Subsequently, the samples were washed with PBS and stained with DAPI for 15 min. The samples were washed again with PBS and images were obtained using an inverted fluorescence microscope (Zeiss, Germany).

2.14.3. DNA quantification. The quantification of doublestranded DNA (dsDNA) was used to investigate the cell proliferation into the developed FS for 1, 3 and 7 days. Initially, cells were lysed by osmotic (by adding ultra-pure water) and thermal shock. The obtained supernatant was analyzed using PicoGreen dsDNA kit (Life Technologies, UK). The recovered supernatant was read on a microplate reader (BioTek, USA) using a 485 and $528 \mathrm{~nm}$ as excitation and emission wavelengths, respectively. The DNA amounts were calculated from a standard curve. At least triplicates were performed for each studied condition.

\subsection{Statistical analysis}

Presented data were expressed as average \pm standard deviation (SD) of at least three replicates. The error bars present in the graphics denote the SD. For AFM and WCA assays, the population was normally distributed and one way Anova followed by Tukey's test were used. Specifically for cellular assays, two way Anova followed by Tukey's test were used. The statistical analysis was performed using the software GraphPad Prism 6.0 for Windows. Statistical significance was accepted for a $\left(^{*}\right) p<$ 0.05 .

\section{Results and discussion}

\subsection{Structural characterization of functionalized graphene}

3.1.1. Raman spectroscopy. Raman spectroscopy is one of the most relevant techniques for the characterization of carbon and graphene based materials. Fig. 1 presents the Raman spectra of (a) pristine EG, f-EG, f-GF (obtained after ultrasonication of the f-EG during $4 \mathrm{~h}$ ) and (b) pristine MWNTs, fMWNTs and f-GNR (obtained after ultrasonication of the fMWNTs during $4 \mathrm{~h}$ ). All the spectra exhibit the three characteristic bands of graphene-based carbon materials (D, G and 2D).

The first peak near $1342 \mathrm{~cm}^{-1}$ (D band) is due to the presence of $\mathrm{sp}^{3}$ carbon atoms, indicating the presence of defects in the hexagonal $\mathrm{sp}^{2}$ carbon lattice of graphene. The second peak near $1580 \mathrm{~cm}^{-1}$ ( $\mathrm{G}$ band) results from in-plane vibration of the ordered $\mathrm{sp}^{2}$ bonded carbon atoms. ${ }^{45,46}$ The intensity ratio between $\mathrm{D}$ and $\mathrm{G}$ peaks (measured as the ratio of the D and $\mathrm{G}$ peak areas, $I_{\mathrm{D}} / I_{\mathrm{G}}$ ) has been used as a method to quantify the increase in disorder caused by covalent chemical modification, ripples, edges and charged impurities. ${ }^{47}$ The third peak near $2700 \mathrm{~cm}^{-1}$ (2D band) has almost the double frequency of the D band and results from the second order Raman scattering. ${ }^{48}$ This peak may be used to determine the number of stacked layers (up to 5) in n-layer graphene, by the analysis of its shape, width and position of the $2 \mathrm{D}$ band.${ }^{47}$ Ferrari et al. ${ }^{49}$ reported that an increase in the number of layers originates a broader $2 \mathrm{D}$ band shifted to higher wavenumber. Pure graphene presents a single sharp 2D peak with higher intensity than the $\mathrm{G}$ band, while in pristine graphite a double structure of the $2 \mathrm{D}$ peak is observed, near $2720 \mathrm{~cm}^{-1}$. $^{\mathbf{4 9 , 5 0}}$ The Raman spectrum of MWNTs exhibits a 2D band shifted to $2683 \mathrm{~cm}^{-1}$ due to the strain induced by the nanotube curvature on the graphene layers. ${ }^{46}$

The analysis of the Raman spectra in Fig. 1a shows that the $\left(I_{\mathrm{D}} / I_{\mathrm{G}}\right)$ for EG increased from 0.24 to 0.49 after functionalization (formation of f-EG), and this ratio further increased to 0.56 after f-EG exfoliation to form f-GF. The $\left(I_{\mathrm{D}} / I_{\mathrm{G}}\right)$ increase may be related to the increase in $\mathrm{sp}^{3}$ carbon relative to the overall $\mathrm{sp}^{2}$ carbon content, due to the functionalization (from EG to f-EG) and then due to the exfoliation process that yields thinner f-GF flakes (from f-EG to f-GF, since only the outer f-EG graphene layers are functionalized). ${ }^{37}$ We also verified an increase in the $\left(I_{\mathrm{D}} / I_{\mathrm{G}}\right)$ ratio after oxidation of EG in a previous work. ${ }^{38}$

The analysis of the Raman spectra in Fig. 1b shows that MWNTs exhibit an inverse behavior, with a decrease of $\left(I_{\mathrm{D}} / I_{\mathrm{G}}\right)$ from 1.38 to 0.95 after functionalization (formation of $\mathrm{f}$ MWNTs) and this ratio further decreased to 0.73 after $\mathrm{f}$ MWNTs exfoliation to form f-GNR. The large $\left(I_{\mathrm{D}} / I_{\mathrm{G}}\right)$ ratio observed for the pristine MWNTs is due to the large fraction of nanotubes containing defects. The functionalization process may select the MWNTs with higher $\mathrm{sp}^{2}$ carbon content, since it is based on a cycloaddition to $\mathrm{sp}^{2}$ carbon, thus forming $\mathrm{f}$ MWNTs with lower $\left(I_{\mathrm{D}} / I_{\mathrm{G}}\right)$ than the overall pristine MWNTs in spite of the covalent functionalization of their surface. In a previous work, a similar decrease in the $\left(I_{\mathrm{D}} / I_{\mathrm{G}}\right)$ ratio was observed after permanganate oxidation of the same grade of 

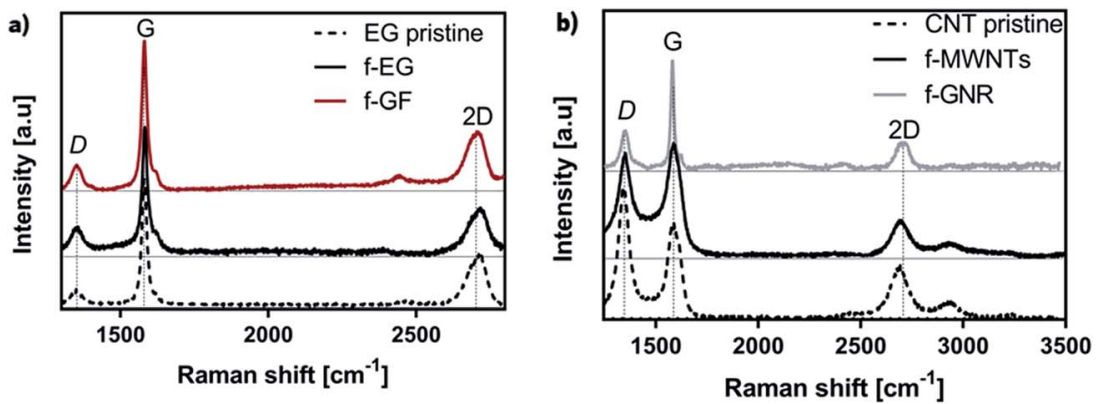

Fig. 1 The Raman spectra obtained of (a) pristine EG, f-EG and f-GF and (b) pristine MWNTs, $f-M W N T s$ and f-GNR.

MWNTs. ${ }^{38}$ The f-GNR that result from unzipping of the outer graphene layer of the f-MWNTs is a two-dimensional material with a Raman spectrum that is similar to that of the graphene derivatives in Fig. 1a.

The downshift of the 2D band from $2718 \mathrm{~cm}^{-1}$ (EG) to 2704 $\mathrm{cm}^{-1}$ (f-GF) and the higher symmetry of this band for f-GF relative to EG and f-EG supports the few-layer nature of f-GF. ${ }^{49}$ Regarding the spectra of f-GNR, the upshift of the 2D band position from $2683 \mathrm{~cm}^{-1}$ (MWNTs) to $2695 \mathrm{~cm}^{-1}$ is consistent with the absence of MWNTs and formation of few-layer functionalized graphene nanoribbons. ${ }^{49}$ Similar results were also obtained by Cunha et al. ${ }^{51}$

3.1.2. Ultraviolet-visible spectroscopy. The UV-Vis spectra of functionalized few-layer graphene (f-GF and f-GNR) suspensions are presented in Fig. 2. The concentration of the functionalized graphene in suspension was determined according to the Lambert-Beer law, considering the weight absorptivity coefficient $(\alpha)$ of $1600 \mathrm{~L} \mathrm{~g}^{-1} \mathrm{~m}^{-1}$ and $1400 \mathrm{~L} \mathrm{~g}^{-1} \mathrm{~m}^{-1}$ for $\mathrm{f}-\mathrm{GNR}$ and f-GF suspensions, respectively, at the wavelength of $250 \mathrm{~nm}$, as was reported by Cunha et al..$^{51}$ and by Xu et al. ${ }^{52}$ The

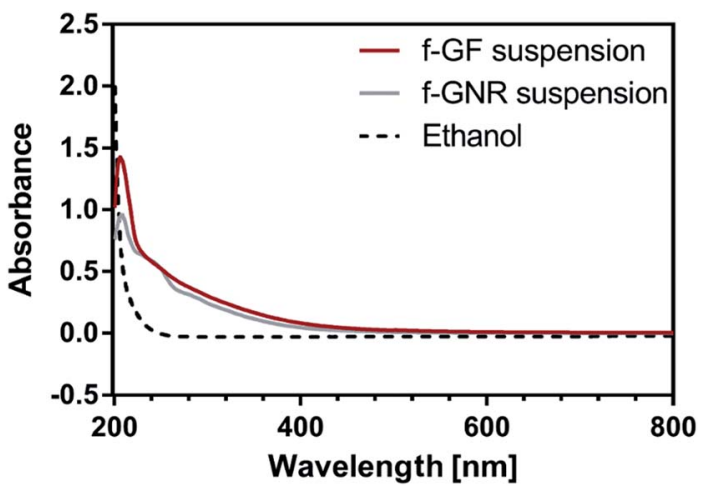

Fig. 2 UV-Vis spectra obtained for $f-G F$ and $f-G N R$ suspensions.
UV-Vis spectra of f-GNR suspension in Fig. 2 exhibits a maximum absorbance at $250 \mathrm{~nm}$. These results are similar to those reported by Cunha $e t a l$. and is consistent with the presence of materials with conjugated carbon-carbon bonds such as graphene derivatives in solution. ${ }^{51}$

Regarding the UV-Vis spectra of f-GF suspension, it also presents a maximum absorbance at $250 \mathrm{~nm}$, attributed to $\pi \rightarrow$ $\pi^{*}$ transitions. $^{53}$

The concentration of the functionalized few-layer graphene suspensions produced are presented in Table 1 , as well as the $\%$ yield of the exfoliation process.

Table 1 shows that both f-EG and f-MWNTs yield suspensions with a reasonable concentration of f-GF and f-GNR. The resultant suspensions of functionalized graphene were concentrated for the production of the FS, which requires large volumes of f-GF and f-GNR with a concentration of $0.25 \mathrm{mg}$ $\mathrm{mL}^{-1}$.

3.1.3. Thermogravimetric analysis. The functionalization of MWNTs and EG by the DCA reaction was evaluated by TGA. fMWNTs and f-EG in powder form were analyzed. The results are presented in Fig. 3.

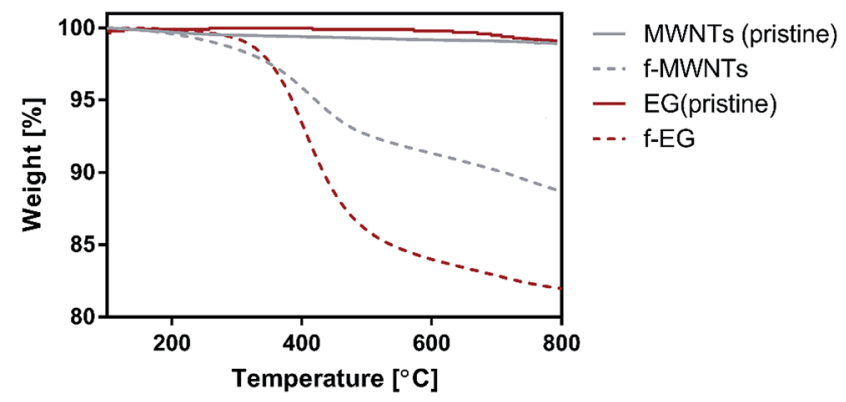

Fig. 3 The TGA curves obtained for pristine EG and f-EG (red lines), and for pristine MWNTs and f-MWNTs (gray lines).

Table 1 Initial concentration, $[C]_{i}$, of $f-E G$ and $f-M W N T s$, concentration, $[C]$, of $f-G F$ and $f-G N R$ suspensions and yield of the exfoliation process

\begin{tabular}{lll}
\hline Suspension & {$[\mathrm{C}]_{\mathrm{i}}$ of functionalized precursor $\left[\mathrm{mg} \mathrm{mL}^{-1}\right]$} & $\begin{array}{l}{[\mathrm{C}] \text { of functionalized }} \\
\text { graphene in suspension }\left[\mathrm{mg} \mathrm{mL}^{-1}\right]\end{array}$ \\
\hline f-GF & 1.000 & 0.037 \\
f-GNR & 1.000 & 0.033
\end{tabular}


The TGA results show that f-MWNTs and f-EG present thermal degradation curves with similar shape. The onset of thermal degradation is observed at a lower temperature for $\mathrm{f}$ MWNTs, however their overall weight loss at $800{ }^{\circ} \mathrm{C}$ is smaller than that of f-EG. The pristine materials, EG and MWNTs, are thermally stable along the temperature range analyzed, as expected for pristine materials with low contamination level. ${ }^{\mathbf{5 4 , 5 5}}$

The weight loss measured for the functionalized materials is due to the thermal degradation of the chemical groups bonded, indicating the successful functionalization of MWNTs an EG. fEG and f-MWNTs exhibit a weight loss of $18.1 \mathrm{wt} \%$ and 11.3 $\mathrm{wt} \%$, respectively, at $800{ }^{\circ} \mathrm{C}$.

\subsection{In situ build-up if the multilayered films}

After characterization of both types of functionalized graphene (f-GNR and f-GF) and since they were stable in suspension, their potential to be assembled by LbL was evaluated. For that, QCM$\mathrm{D}$ was used to monitor in situ the deposition of the various materials layers - Fig. 4 .

The buildup of 6 bilayers is depicted through the variation of the normalized frequency $(\Delta f / \nu)$ and dissipation $(\Delta D)$ of the $3^{\text {rd }}$, $5^{\text {th }}$ and $7^{\text {th }}$ overtone for (CHI/f-GF-ALG) ${ }_{6}$ and (CHI/f-GNR-ALG) $)_{6}$, Fig. $4 \mathrm{a}$ and $\mathrm{b}$, respectively. In both cases, it can be observed that $\Delta f$ decreases with the injection of CHI and f-GF or f-GNR-ALG, which means that the mass of these materials was adsorbed on the surface of the crystal at each deposition step. Comparing both (CHI/f-GF-ALG) $)_{6}$ and (CHI/f-GNR-ALG) $)_{6}$, a strong adsorption was verified for the films containing f-GF. This fact is possibly related to a stronger interaction between f-GF-ALG and CHI when compared with f-GNR-ALG and CHI. Regarding $\Delta D$, it can be observed that is increasing with the injection of $\mathrm{CHI}$ and $\mathrm{f}$-GF or f-GNR-ALG in both cases. $\Delta D$ gives an indirect indication of the film's viscoelastic properties. Thus, such $\Delta D$ increase demonstrates that the assembly of the multilayer film is not rigid and exhibits the typical viscoelastic behavior of polymeric systems. Due to the obtained results, the adsorption behavior does not obey to the Sauerbrey equation, confirming the viscoelastic character of these films.

The experimental results were modulated using the Voigt model, in order to estimate the thickness, shear modulus, and viscosity of the produced films. By assuming $\rho_{\mathrm{s}}=1000 \mathrm{~kg} \mathrm{~m}^{-3}$,

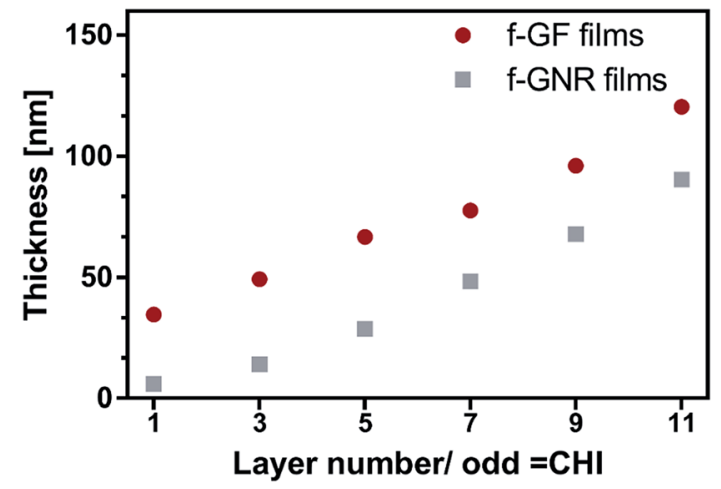

Fig. 5 Evolution of thickness of (๑) (CHI/f-GF-ALG) 6 and ( $($ ) $(\mathrm{CHI} / \mathrm{f}-$ GNR-ALG) ${ }_{6}$ films as a function of the layers deposited calculated based on the Voigt model.

$\eta_{\mathrm{s}}=0.001 \mathrm{~kg} \mathrm{~m}^{-1} \mathrm{~s}^{-1}$ and $\rho_{\mathrm{L}}=1200 \mathrm{~kg} \mathrm{~m}^{-3}$, it was possible to estimate the thickness of the films as a function of adsorbed layers. As expected, Fig. 5 shows that the thickness of both films increases as the adsorbed layers increase. A thickness of $125 \mathrm{~nm}$ and $113 \mathrm{~nm}$ was estimated for (CHI/f-GF-ALG) ${ }_{6}$ and (CHI/f-GNRALG $)_{6}$, respectively. These estimated values are based on the assumption that the adsorbed layers have a uniform thickness.

In addition to the thickness, the viscosity and the shear modulus were also estimated under the same aforementioned assumptions used for thickness - Fig. 6. It can be seen that both properties are increasing while the buildup of the films is occurring for both (CHI/f-GF-ALG) 6 and (CHI/f-GNR-ALG) 6 . These results are similar to those reported in other studies which are related to the deposition of viscoelastic films. ${ }^{41,56}$

\subsection{Scanning electron microscopy}

The morphology of the upper side (last layer produced) of the FS films as well as their cross-section were analyzed by SEM. All the films were easily removed from the PP substrate and demonstrated an easy handling. Only the upper side was evaluated since the lower side, which is in contact with the substrate, was previously reported by us and concluded that leads to smoother surfaces than the upper side of control films ${ }^{57}$ or the upper side of films containing graphene. ${ }^{38}$ a)

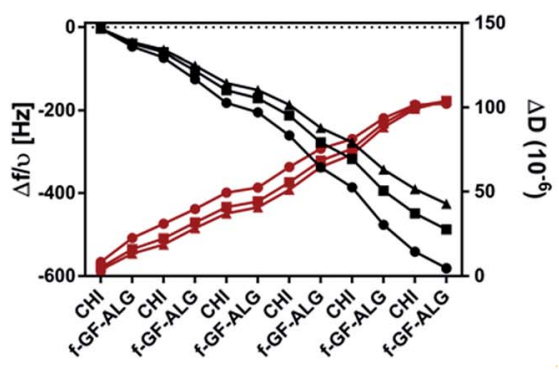

b)

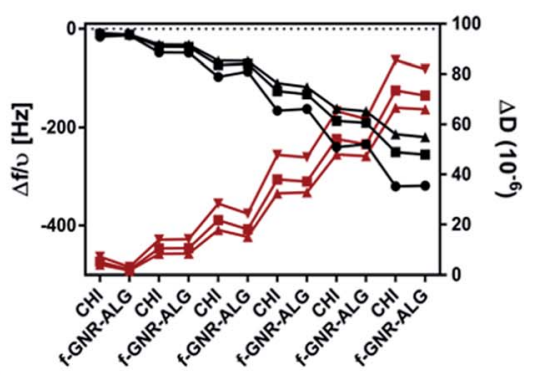

Fig. 4 Normalized frequency $(\Delta f / \nu)$ and dissipation $(\Delta D)$ changes measured by QCM-D during the buildup of (a) $(\mathrm{CHI} / \mathrm{f}-\mathrm{GF}-\mathrm{ALG})_{6}$ and $(\mathrm{b})(\mathrm{CHI} / \mathrm{f}-$ GNR-ALG) 6 . The data presents the $3^{\text {rd }}(\mathbf{\bullet}), 5^{\text {th }}(\boldsymbol{\square})$ and $7^{\text {th }}(\mathbf{\Delta})$ overtones. 
a)

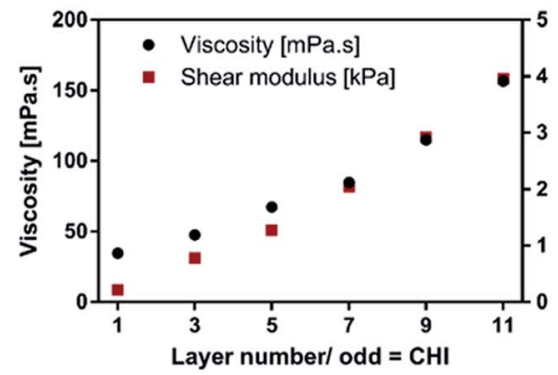

b)

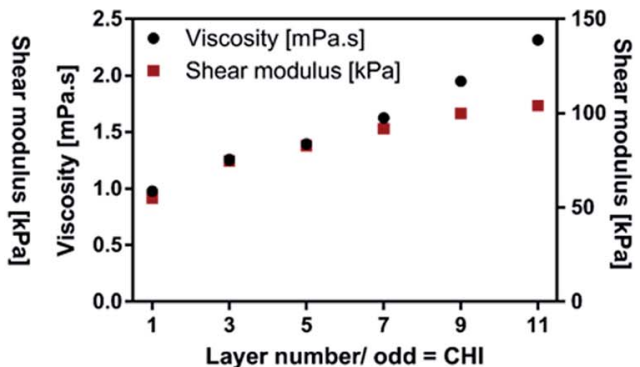

Fig. 6 Viscosity $(\bullet)$ and shear modulus ( $\square)$ of (a) $(\mathrm{CHI} / \mathrm{f}-\mathrm{GF}-\mathrm{ALG})_{6}$ and (b) $(\mathrm{CHI} / \mathrm{f}-\mathrm{GNR}-\mathrm{ALG})_{6}$ film as a function of the layers deposited calculated with the Voigt model.
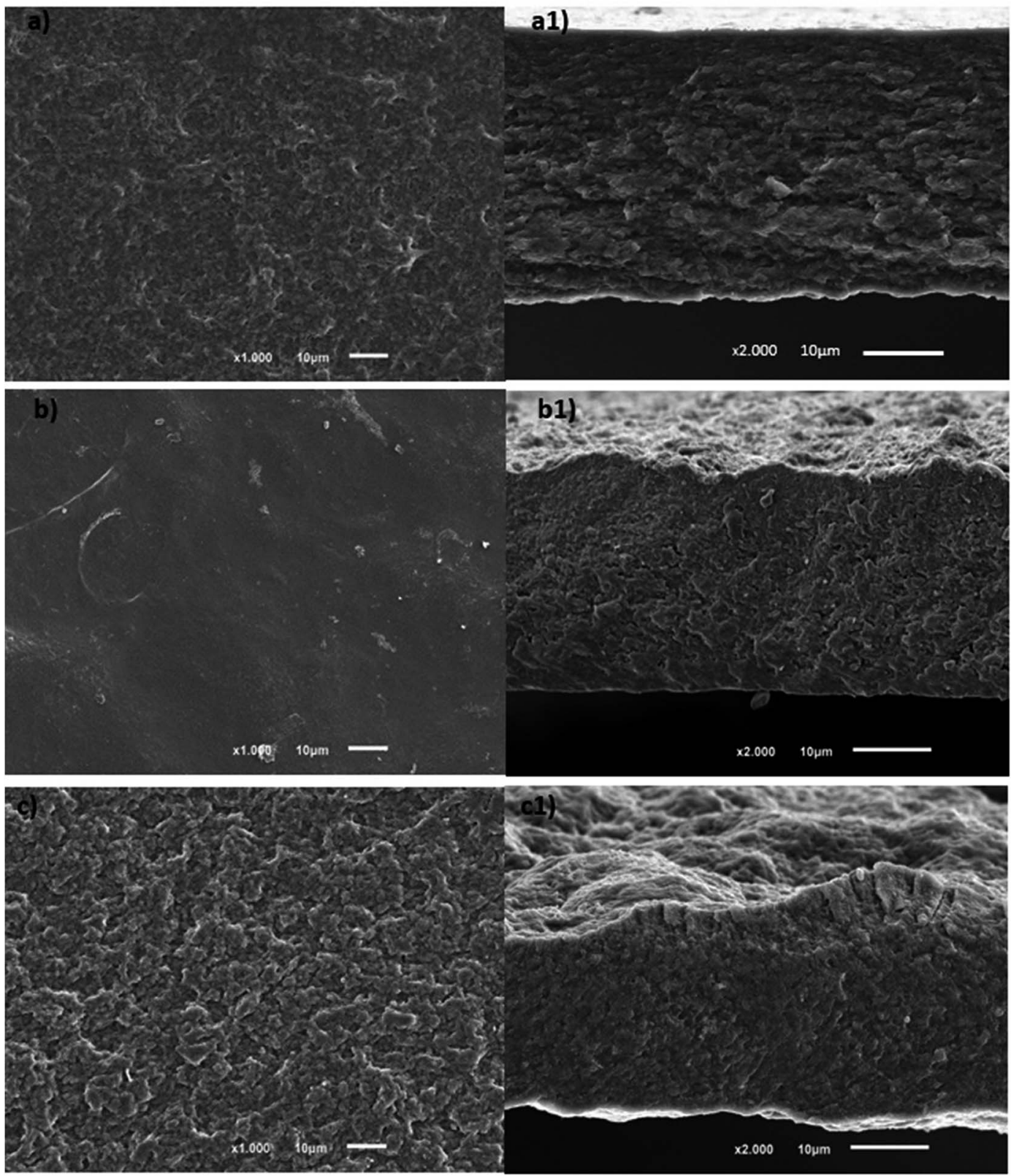

Fig. 7 SEM images of the upper side of the (a) control films, (b) (CHI/f-GF-ALG) 200 and (c) (CHI/f-GNR-ALG) 200. Cross-section of (a1) control; (b1) (CHI/f-GF-ALG) 200 and (c1) (CHI/f-GNR-ALG) 200 FS films. 
The obtained results of the surface morphology of the control film, Fig. 7a, are similar to those reported in a previous work. ${ }^{57}$ At a microscale, comparing $(\mathrm{CHI} / \mathrm{ALG})_{200}$ and $(\mathrm{CHI} / \mathrm{f}-\mathrm{GF}-$ $\mathrm{ALG})_{200}$, it is observed that the addition of $\mathrm{f}-\mathrm{GF}$ resulted in less porous and smoother films - see Fig. 7a and b, as also confirmed by AFM (see Fig. 8). Concerning the FS films with fGNR, Fig. 7c, they show less porosity than the control ones and are rougher, as also confirmed by AFM (see Fig. 8). a)

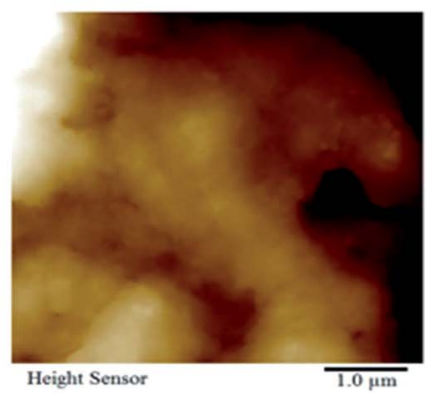

b)

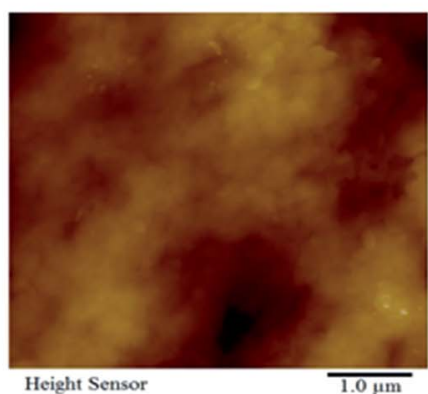

c)

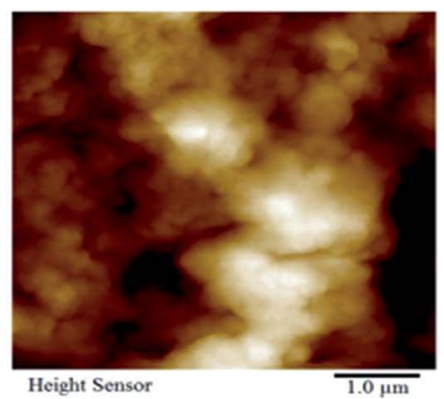

d) a1)
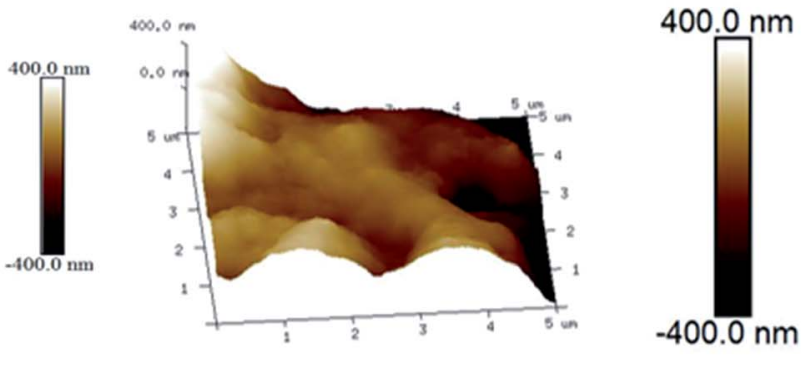

b1)
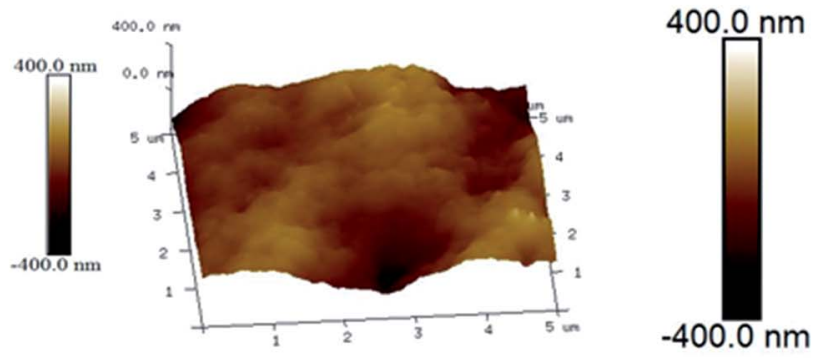

c1)
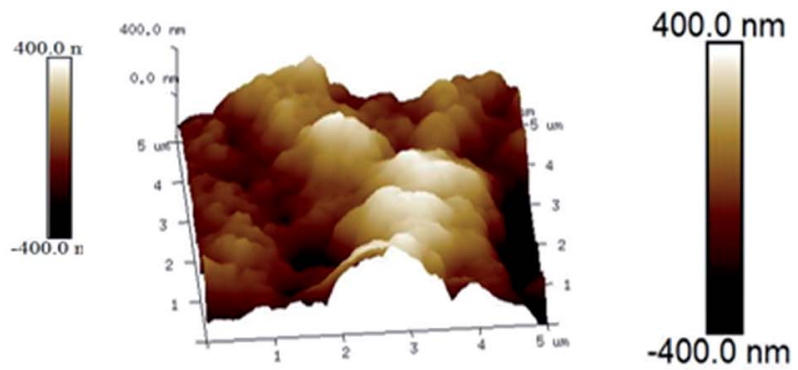

e)

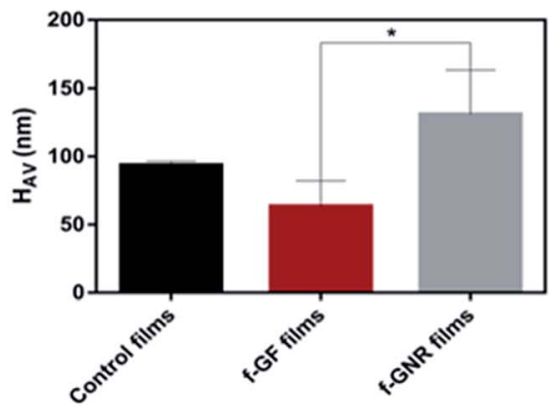

Fig. 8 AFM surface results and images with respective 3D representation of: (a and a1) control FS (b and b1) f-GF FS and (c and c1) f-GNR FS. (d) $R_{\mathrm{RMS}}$ and (e) $H_{\mathrm{AV}}$ of all produced FS. Significant differences were state for $p<0.01(* *)$ and $p<0.05(*)$. 
Concerning the cross-section of graphene films (f-GF and fGNR films), they are represented in Fig. 7b1 and c1, respectively. All cross-sections exhibited wrinkle-like structures. However, this seems to be emphasized by the presence of functionalized graphene, since the control film is more lookalike a layered structure (Fig. 7a1). Besides, the cross-section of f-GF films shows a much denser structure than that of fGNR films. This can be related to a much stronger interaction between CHI and f-GF than CHI and f-GNR, which could result in an enhancement of the mechanical properties, as suggested by Han et al. for $\mathrm{CHI} / \mathrm{GO}$ composites. ${ }^{58}$ Moreover, in the SEM images of both graphene FS films, no exposed f-GF nor f-GNR sheets were found, which accentuates a strong interaction with the surrounding polymers. Such behavior was also found by $\mathrm{Li}$ et al. with genipin-cross-linked $\mathrm{CHI} / \mathrm{GO}$ composites. ${ }^{59}$

All the produced films show some porosity, which is an important characteristic for biomedical applications. Indeed, any scaffold for biomedical applications requires a porous structure for transporting oxygen, nutrients and waste metabolites in and out of the material without compromising its mechanical stability. ${ }^{32,57,60,61}$

\subsection{Atomic force microscopy imaging}

The 2D and 3D surface topographies of the upper side of the films were imaged using AFM - Fig. 8. The root-mean-square deviation values of the surface roughness $\left(R_{\mathrm{RMS}}\right)$ for the region represented in Fig. 8a-c are presented in Fig. 8a.

It was previously reported by us that the substrate side of FS films (in contact with CHI) usually leads to flatter surfaces than their upper side, presenting a roughness of $49 \pm 15 \mathrm{~nm} .{ }^{57}$

Regarding the $R_{\mathrm{RMS}}$ of the upper side of control films, they present a moderate roughness of $117 \pm 2 \mathrm{~nm}$. This moderate roughness may be explained by the significant presence of some polymeric loops and tails, producing rough surface areas. ${ }^{62}$ When f-GF and f-GNR were incorporated into the polymeric matrix, some changes were visualized. Regarding (CHI/f-GF$\mathrm{ALG})_{200}$ films, a decrease in the $R_{\mathrm{RMS}}$ is verified, comparing to the control ones, as visible from Fig. $8 \mathrm{~b}$ and b1, where the FS evidenced a roughness value of $75 \pm 3 \mathrm{~nm}$. This effect may be explained by the flat topography characteristic of graphenebased materials. Opposite results were obtained in a previous work when o-GF with CHI and ALG was used in multilayered FS films. ${ }^{38}$

On the other hand, the (CHI/f-GNR-ALG) $)_{200}$ FS films exhibited the highest $R_{\text {RMs }}$ value of $164 \pm 39 \mathrm{~nm}$, as visible from Fig. $8 \mathrm{c}$ and $\mathrm{c} 1$. This was an expected behavior because, similar to CNTs, nanoribbons easily bend or present a twisted morphology, which may result in bundles structures. ${ }^{62}$ Similar results were obtained with GO by Gudarzi et $a l^{63}$ as well as by us in a previous work, ${ }^{38}$ in which an increase in the $R_{\mathrm{RMS}}$ was verified when o-GNR was used in the polymeric multilayered films.

Considering the potential biomedical application of FS films with f-GF and f-GNR, it should be noted that roughness is related to cell adhesion. Indeed, generally smoother surfaces exhibit lower cell adhesion than rougher surfaces. ${ }^{61}$ Thus, the higher roughness of (CHI/f-GNR-ALG) ${ }_{200}$ films may benefit the cell adhesion and proliferation. Biological assays confirmed this feature (Section 3.11 - Fig. 15). The presence of f-GNR increased the surface roughness and clearly enhanced both the cell adhesion and proliferation of these FS films when compared with the ones with f-GF.

The average height $\left(H_{\mathrm{AV}}\right)$ values are shown in Fig. 8e). The $H_{\mathrm{AV}}$ values range from 55 to $131 \mathrm{~nm}$, being the lowest value for the $(\mathrm{CHI} / \mathrm{f}-\mathrm{GF}-\mathrm{ALG})_{200} \mathrm{FS}$ and the highest one for the $(\mathrm{CHI} / \mathrm{f}-$ GNR-ALG) $)_{200}$ film. It is possible to see that these values are near to the $R_{\mathrm{RMS}}$ ones, which indicates that the roughness is constant along the FS film.

\subsection{Thermogravimetric analysis}

TGA analysis was performed in order to evaluate the thermal stability and the weight composition of the FS films produced. The results are illustrated in Fig. 9a and b. It can be seen that both films with graphene present a similar thermal stability compared with the control one when heated up to $800{ }^{\circ} \mathrm{C}$ under inert atmosphere, which means that the thermal stability is not affected by the presence of functionalized graphene. The initial weight loss below $200{ }^{\circ} \mathrm{C}$ is mostly related to the elimination of strongly adsorbed water and it slightly decreases in the presence of $f-G F$ or f-GNR. The major weight loss was verified in the temperature range from $200{ }^{\circ} \mathrm{C}$ to $360{ }^{\circ} \mathrm{C}$, due to the decomposition of the polymeric materials as well as the functional a)

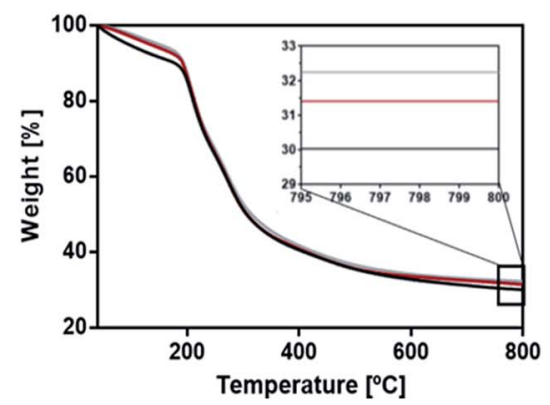

b)

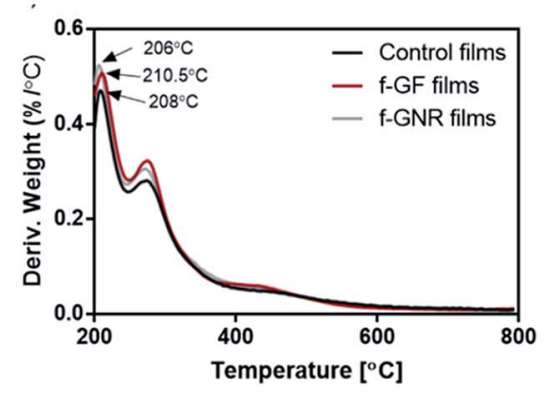

Fig. 9 TGA graphic representation (a) thermogravimetric (TGA) curves with inset showing a magnification for the temperature range of 795$800{ }^{\circ} \mathrm{C}$ and (b) derivative of the weight loss curves (DTGA) for all produced FS, as a function of temperature $\left({ }^{\circ} \mathrm{C}\right.$ ). 
groups of f-GF or f-GNR. Similar behaviors were reported by Wang et al. ${ }^{64}$ for composites with $\mathrm{CHI}$ and graphene derivatives, Ionita et al. ${ }^{65}$ for ALG and GO composites and for CHI/ALG with o-GF or o-GNR composites. ${ }^{38}$ It is observed that the weight loss tends to stabilize above $600{ }^{\circ} \mathrm{C}$, leaving a residual carbonaceous material from the thermal degradation of f-GF, f-GNR and from the polymer carbon backbone. As shown in Fig. 9a, the films containing f-GNR and f-GF present a slightly higher residual weight relative to the control ones. In fact, the incorporated graphene derivatives undergo a small weight loss corresponding to the degradation of the functional groups bonded to their surface, leaving the graphene material intact, which is characterized by high thermal stability under inert atmosphere. The residual weight of the (CHI/f-GF-ALG) $)_{200}$ and (CHI/f-GNR$\mathrm{ALG})_{200}$ films is approximately $31.4 \mathrm{wt} \%$ and $32.2 \mathrm{wt} \%$, respectively. The difference between the residual weight measured from f-GF films and the control films (without graphene) was approximately $1.40 \mathrm{wt} \%$ while for f-GNR films, this difference was about $2.20 \mathrm{wt} \%$. Considering that, at $800{ }^{\circ} \mathrm{C}$, f-EG and f-MWNTs presented a residual weight of about $81.9 \mathrm{wt} \%$ and $88.7 \mathrm{wt} \%$ respectively (Fig. 3), the weight content of f-GF and f-GNR in the FS was estimated to be approximately 1.7 wt $\%$ and $2.5 \mathrm{wt} \%$, respectively.

\subsection{Water contact angle measurements}

In order to evaluate the wettability of the surfaces of all produced FS films, water contact angle measurements were performed. Only the upper side of each condition was evaluated - see Fig. 10. Regarding the control film, the upper side corresponds to ALG side and shows a WCA of $103 \pm 0.6^{\circ}$, i.e. around $100^{\circ}$. Similar results were also obtained in a previous work for $\mathrm{CHI} / \mathrm{ALG}$ films. ${ }^{32}$ Comparing the upper side of the (CHI/f-GFALG $)_{200}$ and (CHI/f-GNR-ALG) $)_{200}$ FS, they present WCA values of $95.3 \pm 0.4^{\circ}$ and $92.7 \pm 0.7^{\circ}$, respectively, which means that the presence of both functionalized graphene flakes and graphene nanoribbons confers significant differences in terms of wettability when compared to the control film. Indeed, both f-GF

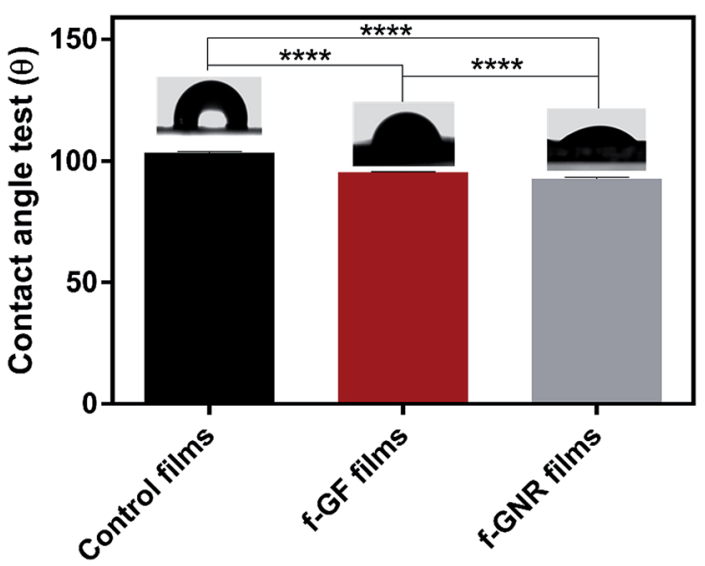

Fig. 10 Water contact angle measurements with representative images for the upper side of each produced condition. Significant differences were state for $p<0.0001(* * * *)$. films and f-GNR films show a decrease in the WCA, which means that both FS films although remaining hydrophobic, they became more hydrophilic when functionalized graphene was added. This behavior was also verified by other works. ${ }^{66}$ Eventually, the functional groups of modified graphene or even the surface morphology turn these FS films more hydrophilic than the control film. ${ }^{66}$

Regarding the down side of FS films, in all cases, is the same material, CHI, so the same behavior is expected. The WCA of CHI side was already extensively reported and presents a WCA around $100^{\circ}$. In a previous study, we already reported a WCA of $100^{\circ}$ in $\mathrm{CHI} / \mathrm{ALG}$ multilayered films, too. ${ }^{32}$

\subsection{Swelling ability studies}

The swelling behavior of the produced FS was evaluated during $48 \mathrm{~h}$ in PBS at $37{ }^{\circ} \mathrm{C}$. The obtained results are illustrated in Fig. 11. Regarding the control films, the results show that they attained the equilibrium after $5 \mathrm{~h}$, reaching a maximum swelling of approximately $1028 \%$. On the other hand, it is verified that the incorporation of both forms of functionalized graphene in the polymeric matrix reduces the water uptake (WU). Besides, more time is necessary to achieve the WU equilibrium, especially for the FS films containing f-GF. Similar results were obtained in multilayered FS films based on GO with ALG and CHI. ${ }^{38}$ Both f-GF and f-GNR films attained a maximum swelling of about $707 \%$ and $571 \%$, respectively. This behavior may be related to the strong interfacial adhesion between the functionalized graphene and the polymeric matrix, which results in lower space for water storage capacity and diffusion, as was suggested by other authors. ${ }^{59,65}$ Since the WU decreases, these strong interactions between functionalized graphene and natural polymers seem to have more impact in their swelling ability than the hydrophilic nature of multilayered films. So, the incorporation of both types of functionalized graphene in $\mathrm{CHI}$ / ALG films seems to be an adequate strategy to control the swelling behavior, replacing the cross-linking methods. This behavior turns graphene-based composites promisors for application in heart valves or skin scaffolds as suggested in literature. $^{59}$

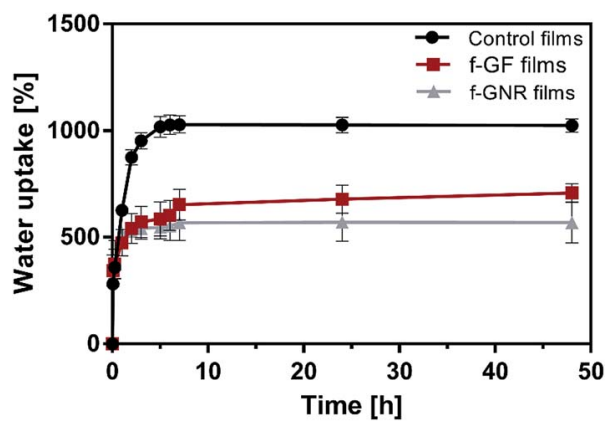

Fig. 11 Variation of the water uptake ability as a function of time of control films (black round symbol), f-GF films (red square symbol) and $\mathrm{f}$-GNR films (gray triangle symbol) in PBS at $37^{\circ} \mathrm{C}$ during a period of $48 \mathrm{~h}$. 


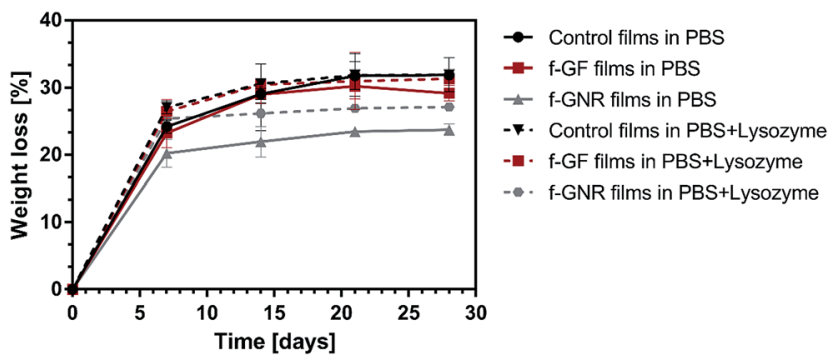

Fig. 12 Degradation behavior of control films (black line), f-GF films (red line) and f-GNR films (gray line) when immersed in PBS and PBS with lysozyme over a period of 28 days at $37^{\circ} \mathrm{C}$.

\subsection{Degradation studies}

When a biomaterial is implanted in the human body the degradation rate of this biomaterial is one of the most important features, since the formation of the new tissue should match with the degradation of the material. Therefore, the biomaterial must be degrade only after appropriately completing its function. ${ }^{67,68}$ The degradation behavior of all produced FS films were assessed and are presented in Fig. 12.

The weight-loss of f-GF, f-GNR and control films was caused by non-enzymatic hydrolysis and enzyme-catalysed hydrolysis, which are two common degradation processes that occur in natural polymers. ${ }^{67,68}$ For the enzyme-catalyzed hydrolysis evaluation a lysozyme solution was used, which is the main enzyme involved in the degradation of chitin derivatives such as CHI. It is present in human serum in concentrations around 7-13 mg $\mathrm{L}^{-1}{ }^{-69}$ For convenience, the maximum concentration was employed during this study.

Fig. 12 presents the degradation behavior of f-GF, f-GNR and control films when immersed in both PBS and PBS + lysozyme during 7, 14, 21 and 28 days.

Independently of the solution used, the degradation was more pronounced in the first seven days for all the studied films. At 7 days, when immersed in PBS medium, it was observed that the control film reached a higher weight loss than the other formulations. The weight loss was around $24 \%, 23 \%$ and $20 \%$ for the control film, (CHI/f-GF-ALG) $)_{200}$ and $(\mathrm{CHI} / \mathrm{f}-$ GNR-ALG $)_{200}$ FS, respectively. Moreover, a higher weight loss was verified for the same period when the samples were incubated in the PBS + lysozyme solution where a value of $27 \%, 26 \%$ and $25 \%$ was verified for the control film, (CHI/f-GF-ALG) ${ }_{200}$ and (CHI/f-GNR-ALG $)_{200} \mathrm{FS}$, respectively. According to Justin et al. ${ }^{70}$ this major initial degradation in PBS + lysozyme may be attributed to the lysozyme reaction to the abundant $\mathrm{CHI}$ chains containing at least 3 acetyl units.

After 7 days of degradation in both solutions, it is clearer the higher weight loss of the control films, comparing to the film with functionalized graphene. At the end of the degradation study, 28 days, a weight loss of about $32 \%, 29 \%$ and $24 \%$ was achieved for the control film, (CHI/f-GF-ALG) $)_{200}$ and (CHI/fGNR-ALG) $)_{200}$ FS, respectively with samples immersed in PBS. For the same period of time but with the samples soaked in PBS + lysozyme solution, a weight loss of about $32 \%, 31 \%$ and $27 \%$ was found for the control FS, (CHI/f-GF-ALG) ${ }_{200}$ and (CHI/fGNR-ALG) ${ }_{200}$ FS, respectively. In general, it was verified that the control film presented a higher weight loss than the films containing f-GF and f-GNR, either in PBS or PBS + lysozyme medium. These results indicate that the incorporation of f-GF and f-GNR seems to induce a greater resistance to degradation. This behavior may be related with the water uptake capability of the films. Indeed, f-GF and f-GNR films present lower capability to swell and lower quantities of PBS and enzyme enter into the polymeric matrix, which results in lower weight loss, either by hydrolysis or by enzyme-hydrolysis action. ${ }^{59} \mathrm{Li}$ et al. also observed a greater resistance to enzymatic degradation by increasing GO content. ${ }^{59}$

\subsection{Dynamical mechanical analysis}

DMA is an adequate technique for characterizing the mechanical/viscoelastic properties of biomaterials since it can use test conditions that closely simulate the physiological environment. ${ }^{32}$

DMA experiments were performed in order to evaluate the effects of the incorporation of functionalized graphene on the mechanical/viscoelastic properties of CHI/ALG FS films. Fig. 13a) presents the variation of the storage modulus, $E^{\prime}$ along the frequency, from 0.1 to $1 \mathrm{~Hz}$ (physiological frequencies). For all produced films, it is verified that $E^{\prime}$ increases with frequency, which is a particular behavior of viscoelastic materials. ${ }^{71}$ Such behavior was already verified in previous works for the control film. ${ }^{72,73}$ The incorporation of $\mathrm{f}-\mathrm{GF}$ and $\mathrm{f}-\mathrm{GNR}$ resulted in an increase of their stiffness which is a typical behavior verified in other studies regarding graphene-reinforced composites with $\mathrm{CHI},{ }^{74} \mathrm{ALG}^{75}$ and with a CHI/ALG matrix ${ }^{38}$ in which it was found that, even for an amount of graphene as low as $0.1 \mathrm{wt} \%$ an increase in stiffness of approximately 2 times was verified. In this work, such increase in stiffness is more marked for the films containing f-GF. Indeed, Fig. 13a shows that the addition of f-GF results in a higher $E^{\prime}$, reaching values 6.6 times higher than the control film while the addition for f-GNR generated values 3.8 times higher than the control films. Similar results were obtained in a previous work with FS films composed of $\mathrm{CHI}, \mathrm{ALG}$ and GO. ${ }^{38}$ In the water uptake experiments it was verified that the control films had the higher ability to swell (about 1000\%), in which more water was able to enter in the polymeric matrix leading to molecular mobility and thus, softening the films. Although a higher water uptake was verified for the (CHI/f-GF-ALG) 200 (about $700 \%$ ) than the (CHI/f-GNRALG) ${ }_{200}$ FS (about $600 \%$ ), it is verified that the former ones are stiffer. This could be ascribed to the less porous structure of (CHI/f-GF-ALG $)_{200}$ films as verified by SEM.

The loss factor, $\tan \delta$ it is the ratio of the amount of energy dissipated by viscous mechanisms relative to energy stored in the elastic component. It is a measure of energy loss and provides information about the damping properties of the films. ${ }^{76}$ The variation of $\tan \delta$ as a function of the frequency is presented in Fig. 12b. Overall, all the produced FS flms present the same behavior. (CHI/f-GNR-ALG) ${ }_{200}$ films exhibited slightly higher $\tan \delta$ values, indicating that such films possess a slightly 
a)

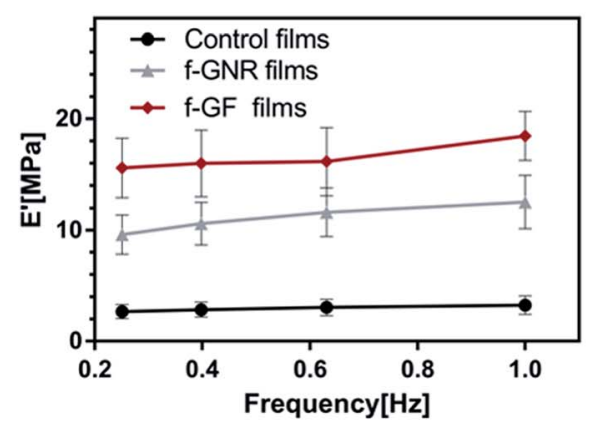

b)

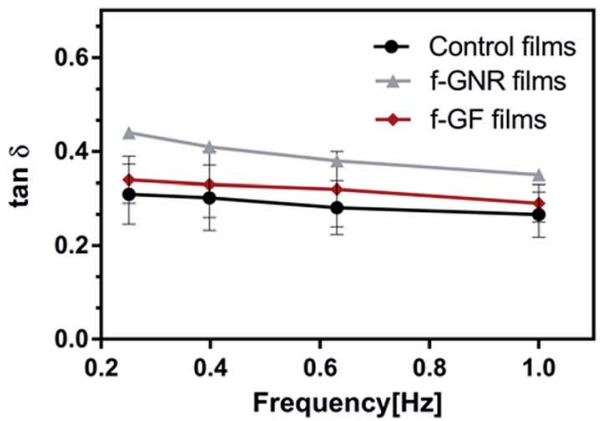

Fig. 13 DMA experiments: variation of (a) storage modulus and (b) loss factor as a function of the frequency, ranging from $0.1-1 \mathrm{~Hz}$, at $37^{\circ} \mathrm{C}$ of $\mathrm{f}-$ GF films, f-GNR films and control films while immersed in PBS to closely simulate the physiological conditions.

higher capacity to dissipate energy. This could be the result of the larger area covered by the interface between the polymeric matrix and the particles for the former composite, that could contribute more for the dissipation of mechanical energy. ${ }^{76}$

$\tan \delta$ exhibit higher values at low frequencies, and close to 0.3 around $1 \mathrm{~Hz}$ (biological frequency) thus, indicating the viscoelastic character of the produced FS films. DMA and specifically this parameter are important when considering the potential of the developed FS films for biomedical application. A biomaterial is subjected to periodic loads in many situations, for example, the biomaterials for cardiovascular applications experiment the pulsed blood pressure. Besides, the viscoelastic character of these films has particular interest since living tissues and their biological constituents also exhibit a viscoelastic behavior at certain stimuli, when subjected to mechanical solicitations. ${ }^{77}$

\subsection{Electrical resistivity measurements}

The electrical resistivity of the FS films was determined by measuring the current that passes through the control film and the films containing f-GF and f-GNR (volume resistivity) in the voltage range between -5.0 and $5.0 \mathrm{~V}$, see Fig. 14. It was observed that the incorporation of $\mathrm{f}-\mathrm{GF}$ and $\mathrm{f}-\mathrm{GNR}$ in the

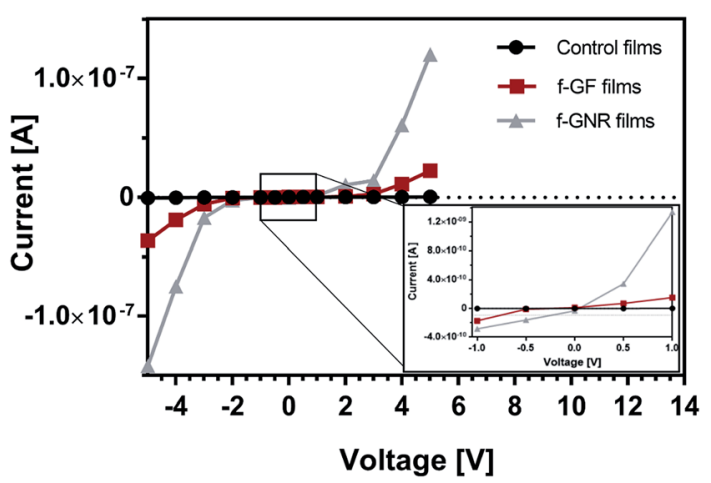

Fig. $14 \quad I-V$ curves obtained for the control, $f-G F$ and $f$-GNR films between $-5.0 \mathrm{~V}$ and $5.0 \mathrm{~V}$ with inset showing a magnification for voltages between $-1.0 \mathrm{~V}$ and $1.0 \mathrm{~V}$. polymeric matrix led to a decrease in the electrical resistivity from $3.00 \times 10^{12} \Omega \mathrm{m}$ to $1.66 \times 10^{10} \Omega \mathrm{m}$ and $2.25 \times 10^{9} \Omega \mathrm{m}$, respectively. Several studies report a decrease of electrical resistivity in composites with graphene or graphene derivatives as fillers. ${ }^{78-82}$ For instance, Li and co-workers reported a similar trend for the resistivity of $\mathrm{GO} /$ polystyrene composites containing $\approx 2 \mathrm{wt} \%$ and $2.5 \mathrm{wt} \%$ of GO. ${ }^{83}$

The structure of the films produced is obtained by the deposition of two polymer layers in which one of them is combined with graphene. The low graphene content and the layered morphology, where an insulating polymer alternates with a polymer/graphene composite, limits the volume resistivity measured perpendicular to the layer direction. Although the f-GF and f-GNR containing FS films still present a resistive behavior, their electrical resistivity decreased by two and three orders of magnitude, respectively, which is a promising indication for the production of conductive films with higher graphene content.

\subsection{Biological assays}

The cytocompatibility of the produced FS films was evaluated through in vitro culture studies by using L929 fibroblasts cells during 1,3 and 7 days.

To understand how cells adhered to the films and to observe their morphology, a DAPI-phalloidin test was performed - see Fig. 15. Comparing all the formulations, it can be noticed that cells behave quite differently for each condition. Although presenting a round shape on day 1 , the cells started to adhere to the FS surface. Comparing all the formulations, the (CHI/f-GNRALG) $)_{200}$ films and TCPS presented more cells attached on its surface than the (CHI/f-GF-ALG) ${ }_{200}$ films and the control ones. After 3 days, although an increase in cell number was verified for all conditions, the (CHI/f-GF-ALG) ${ }_{200}$ films were the ones where the increase was not pronounced. Regarding the cell morphology, cells are better spread and revealed an elongated shape morphology for the (CHI/f-GNR-ALG) $)_{200}$ FS films and TCPS. In the case of (CHI/f-GF-ALG $)_{200}$ films, the cells were more round than the other conditions and, the cells on the surface of the control film even formed some agglomerates. At day 7, the cell density increased for all conditions studied. Some cells on 
Day 1
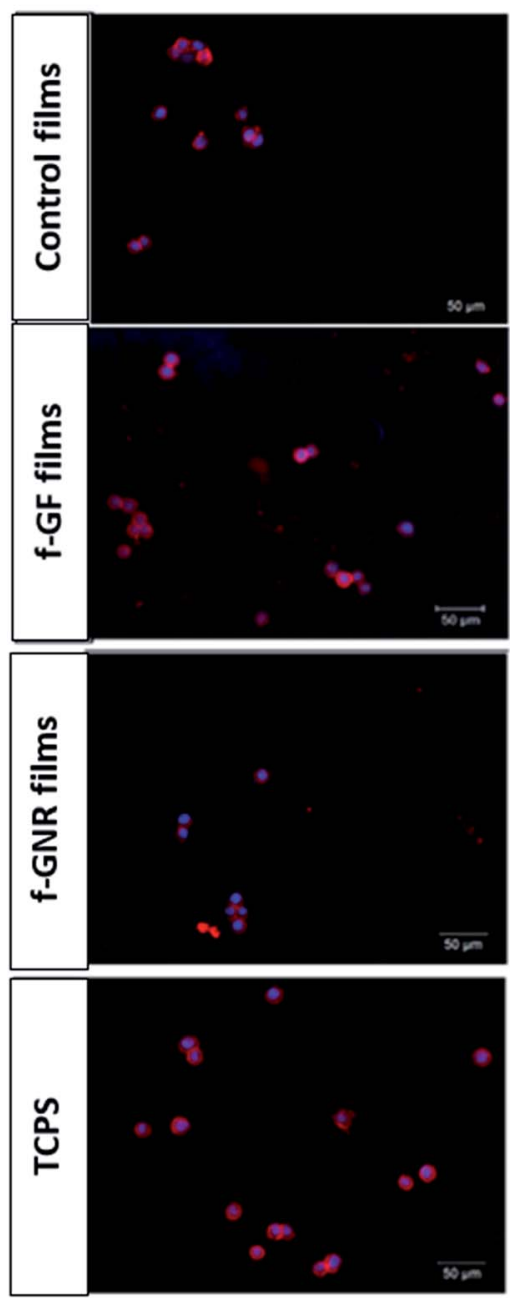

Day 3
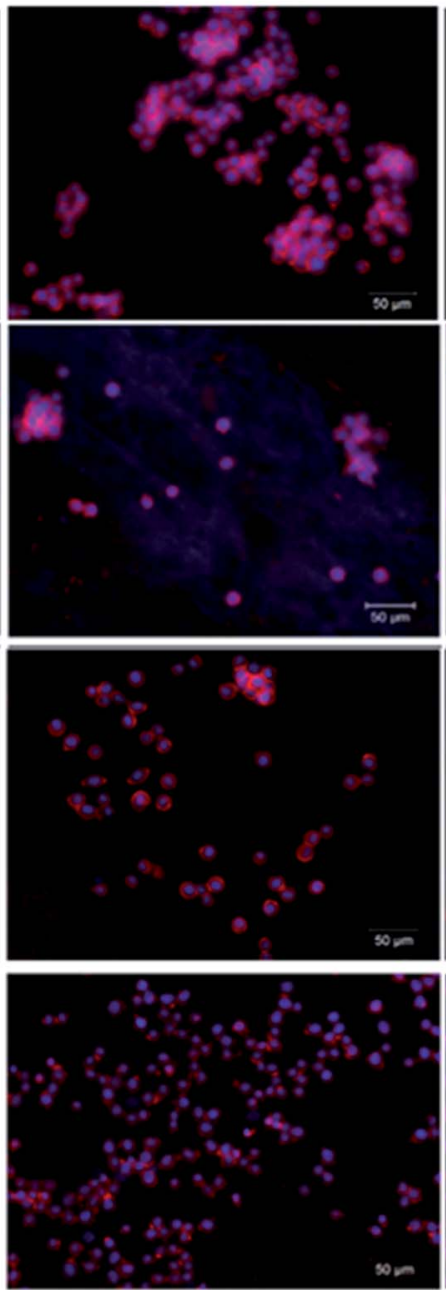

Day 7
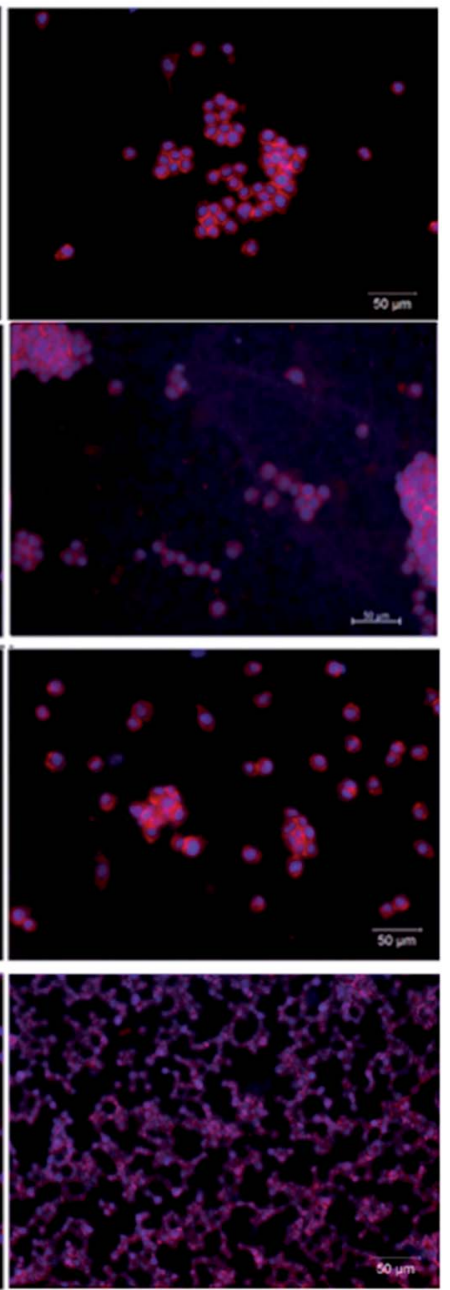

Fig. 15 Evaluation of cells' morphology using DAPI for nuclei staining (blue labelled) and phalloidin for F-actin filaments staining (red labelled) by fluorescence microscopy at 1,3 , and 7 days post-seeding on control films, $\mathrm{f}$-GF films, $\mathrm{f}$-GNR films and TCPS. The scale bar is $50 \mu \mathrm{m}$ and is the same for all images.

the control and on the f-GF film start to change their shape morphology, being more elongated. Regarding the cell morphology of the (CHI/f-GNR-ALG) $)_{200}$ films, they revealed a comparable morphology and behavior with TCPS. Cell attachment to hydrophilic surfaces occurs more readily and more strongly than to hydrophobic ones. ${ }^{84}$ The increase in surface roughness and the subsequent increase in surface area are important features to enhance cell attachment, independently of the cell type as was verified in other previous work. ${ }^{85}$ Indeed the surface of (CHI/f-GNR-ALG) ${ }_{200}$ films presents lower water contact angle and higher roughness than that of $(\mathrm{CHI} / \mathrm{f}-$ GF-ALG) $)_{200}$ films.

DNA quantification was used to assess the cell proliferation over a period of 7 days and the results are presented in Fig. 16. All the tested conditions presented an increase in cell proliferation, over the time period of the experiments. At day 1, significant differences were found between TCPS and both control and f-GNR films $(p>0.01(* *))$. In contrast no significant

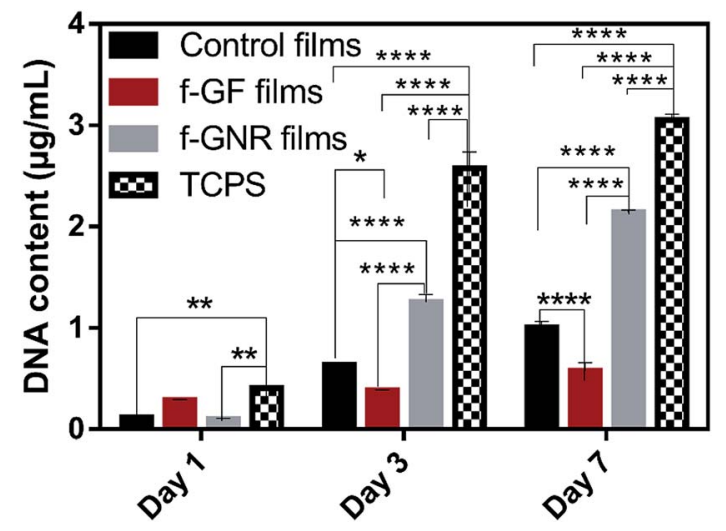

Fig. 16 Cellular proliferation through the determination of the DNA content (DNA quantification assay) for 1,3 and 7 days for (CHI/ALG) 200 , $(\mathrm{CHI} / \mathrm{f}-\mathrm{GF}-\mathrm{ALG})_{200}$ and $(\mathrm{CHI} / \mathrm{f}-\mathrm{GNR}-\mathrm{ALG})_{200}$. Significant differences were found for $p>0.05(*) ; p>0.01(* *) ; p>0.001(* *)$ and $p>$ $0.0001(* * * *)$. 
differences were found between TCPS and (CHI/f-GF-ALG) $)_{200}$ films. At day 3, significant differences were found for TCPS in relation to all the others conditions $(p>0.0001(* * * *))$. Besides, for the same period of time, $(\mathrm{CHI} / \mathrm{f}-\mathrm{GNR}-\mathrm{ALG})_{200}$ films exhibited significant increase in relation to both control and f-GF FS $(p>$ $0.0001(* * * *))$. Also, the control films proliferated more than the $(\mathrm{CHI} / \mathrm{f}-\mathrm{GF}-\mathrm{ALG})_{200}$. At day 7, TCPS exhibited the same differences as the ones verified at day 3. In addition, f-GNR films presented significant differences when compared with both control and f-GF films $(p>0.0001(* * * *))$. It was also verified that the $\mathrm{L} 929$ cells proliferated more on the control film than on the $(\mathrm{CHI} / \mathrm{f}-\mathrm{GF}-\mathrm{ALG})_{200}$ film.

Overall, the biological assays demonstrated that the presence of f-GF and f-GNR in the polymeric matrix lead to different effects on L929 cell behavior. When f-GNR is present, an enhancement in terms of adhesion and cell proliferation was verified comparing to both control and f-GF films. Overall, the presence of both f-GF and f-GNR in the polymeric matrix enhanced the biological behavior of L929 cells. The better cytocompatibility of the (CHI/f-GNR-ALG) $)_{200}$ FS films may be related to their lower hydrophobicity and higher roughness. Other works reported an enhanced cell behavior when graphene were included in the polymeric matrix..$^{38,82,86}$

\section{Conclusions}

$(\mathrm{CHI} / \mathrm{f}-\mathrm{GF}-\mathrm{ALG})_{200}$ and (CHI/f-GNR-ALG $)_{200}$ FS films were successfully produced through LbL assembly. The incorporation of f-GF and f-GNR imparts distinctive properties relative to the control FS films. TGA analysis estimates an incorporation of approximately $1.7 \mathrm{wt} \%$ of $\mathrm{f}-\mathrm{GF}$ and $2.5 \mathrm{wt} \%$ of $\mathrm{f}-\mathrm{GNR}$ in the FS films. Both FS films presented lower porosity than the control ones. The incorporation of f-GF led to FS films with lower roughness while an opposite effect was observed with the incorporation of f-GNR. Both graphene reinforced FS films revealed the enhancement of the storage modulus, the $(\mathrm{CHI} / \mathrm{f}-$ GF-ALG) ${ }_{200}$ FS films reaching the higher stiffness. A decrease in water contact angle was observed with the incorporation of f-GF and f-GNR, although both films remain hydrophobic. Besides, the resistance to degradation by hydrolysis and enzymatic action was enhanced in both FS films containing the functionalized graphene while the swelling ability decreased. Electrical measurements revealed that the incorporation of 1.7 and 2.5 wt $\%$ of $\mathrm{f}-\mathrm{GF}$ and f-GNR, respectively, in the (CHI/f-GF$\mathrm{ALG})_{200}$ and (CHI/f-GNR-ALG) ${ }_{200}$ FS films induced a decrease of the electrical resistivity by two and three orders of magnitude, respectively. The biological assays with $\mathrm{L} 929$ cells revealed that all the produced FS films were cytocompatible and, in particular, the L929 were able to proliferate more on the surface of the $(\mathrm{CHI} / \mathrm{f}-\mathrm{GNR}-\mathrm{ALG})_{200}$ films.

The results obtained in this work demonstrate the production of FS films containing graphene derivatives, (CHI/f-GF$\mathrm{ALG})_{200}$ and (CHI/f-GNR-ALG $)_{200}$, with promising properties for biomedical applications such as wound healing and tissue engineering of bone and cardiac tissues.

\section{Acknowledgements}

The authors acknowledge the Portuguese Foundation for Science and Technology (FCT) and the European program FEDER/COMPETE for the financial support through project LA ICVS/3Bs - 2015-2017, and for project PEst-C/CTM/LA0025/ 2013 (LA 25 - 2015-2017). This work was also financially supported by FCT through the scholarships SFRH/BPD/96797/2013 granted to Sofia G. Caridade, SFRH/BD/97606/2013 granted to Maria P. Sousa, and SFRH/BD/87214/2012 granted to Eunice Cunha.

\section{References}

1 E. P. Randviir, D. A. C. Brownson and C. E. Banks, Mater. Today, 2014, 17, 426-432.

2 P. Avouris and C. Dimitrakopoulos, Mater. Today, 2012, 15, 86-97.

3 T. Kuila, S. Bose, A. K. Mishra, P. Khanra, N. H. Kim and J. K. Lee, Prog. Mater. Sci., 2012, 57, 1061-1105.

4 K. S. Novoselov, S. V. Morozov, T. M. G. Mohinddin, L. A. Ponomarenko, D. C. Elias, R. Yang, I. I. Barbolina, P. Blake, T. J. Booth, D. Jiang, J. Giesbers, E. H. Hill and A. K. Geim, Phys. Status Solidi B, 2007, 244, 4106-4111.

5 C. Soldano, A. Mahmood and E. Dujardin, Carbon, 2010, 48, 2127-2150.

6 D. Galpaya, M. Wang, M. Liu, M. Motta, E. Waclawik and C. Yan, Graphene, 2012, 1, 30-49.

7 K. S. Novoselov, V. I. Fal'ko, L. Colombo, P. R. Gellert, M. G. Schwab and K. Kim, Nature, 2012, 490, 192-200.

8 S. Goenka, V. Sant and S. Sant, J. Controlled Release, 2014, 173, 75-88.

9 A. M. Pinto, I. C. Gonçalves and F. D. Magalhães, Colloids Surf., B, 2013, 111, 188-202.

10 H. Bai, C. Li and G. Shi, Adv. Mater., 2011, 23, 1089-1115.

11 Y. Zhang, T. R. Nayak, H. Hong and W. Cai, Nanoscale, 2012, 4, 3833-3842.

12 S. P. Lonkar, Y. S. Deshmukh and A. A. Abdala, Nano Res., 2015, 8, 1039-1074.

13 C. Zhang and T. Liu, Chin. Sci. Bull., 2012, 57, 3010-3021.

14 R. Araújo, M. C. Paiva, M. F. Proença and C. J. R. Silva, Compos. Sci. Technol., 2007, 67, 806-810.

15 M. C. Paiva, F. Simon, R. M. Novais, T. Ferreira, M. F. Proença, W. Xu and F. Besenbacher, ACS Nano, 2010, 4, 7379-7386.

16 M. C. Paiva, E. Cunha, E. P. Freitas, H. Rocha, M. F. Proença, N. M. Alves, J. F. Mano, M. Melle-Franco, F. L. Deepak, M. González-Debs and P. E. Lopes, presented in part at Carbon Materials for Ubiquitous and Sustainable Life, Korea, June, 2014.

17 V. Georgakilas, K. Kordatos, M. Prato, D. M. Guldi, M. Holzinger and A. Hirsch, J. Am. Chem. Soc., 2002, 124, 760-761.

18 M. Paiva, W. Xu, M. F. Proença, R. M. Novais, E. Lægsgaard and F. Besenbacher, Nano Lett., 2010, 10(5), 1764-1768. 
19 V. Georgakilas, M. Otyepka, A. B. Bourlinos, V. Chandra, N. Kim, K. C. Kemp, P. Hobza, R. Zboril and K. S. Kim, Chem. Rev., 2012, 112(11), 6156-6214.

20 T. Kuila, S. Bhadra, D. Yao, N. M. Kim, S. Bose and J. H. Lee, Prog. Polym. Sci., 2010, 35(11), 1350-1375.

21 A. Sionkowska, Prog. Polym. Sci., 2011, 36(9), 1254-1276.

22 M. Rinaudo, Prog. Polym. Sci., 2006, 31(7), 603-632.

23 F. Croisier and C. Jérôme, Eur. Polym. J., 2013, 49(4), 780792.

24 M. Akili, M. Rafatullah, B. Salamatinia, A. Z. Abdullah, M. H. Ibrahim, K. B. Tan, Z. Gholami and P. Amouzgar, Carbohydr. Polym., 2014, 113, 115-130.

25 T. Zerzopoulou, G. Z. Kyzas and D. N. Bikiaris, Materials, 2015, 652-683.

26 I. Y. Kim, S. J. Seo, H. S. Moon, M. K. Yoo, I. Y. Park, B. C. Kim and C. S. Cho, Biotechnol. Adv., 2008, 26(1), 1-21.

27 K. Y. Lee and D. J. Mooney, Prog. Polym. Sci., 2012, 37(1), 106126.

28 D. S. Morais, M. A. Rodrigues, T. I. Silva, M. A. Lopes, M. Santos, J. D. Santos and C. M. Botelho, Carbohydr. Polym., 2013, 95(1), 134-142.

29 J. Borges and J. F. Mano, Chem. Rev., 2014, 114(18), 88838942.

30 R. R. Costa and J. F. Mano, Chem. Soc. Rev., 2014, 43, 34533479.

31 C. Picart, F. Caruso, J. Voegel and G. Decher, Layer-by-layer films for Biomedical Application, John Wiley \& Sons, 2014.

32 J. M. Silva, S. G. Caridade, N. M. Oliveira, R. L. Reis and J. F. Mano, J. Mater. Chem. B, 2015, 3, 4555-4568.

33 I. Armentano, M. F. Dottori, E. Fortunati, S. Mattioli and J. M. Kenny, Polym. Degrad. Stab., 2010, 95(11), 2126-2146.

34 K. Hu, D. D. Kulkarni, I. Choi and V. V. Tsukruk, Prog. Polym. Sci., 2014, 39, 1934-1972.

35 W. K. Chee, H. N. Lim, N. M. Huang and I. Harrison, $R S C$ $A d v .$, 2015, 5, 68014-68051.

36 M. M. Barsan, M. David, M. Florescu, L. T3ugulea and C. M. Brett, Bioelectrochemistry, 2014, 99, 46-52.

37 L. Tang, X. Li, D. Du and C. He, Prog. Nat. Sci.: Mater. Int., 2012, 22(4), 341-346.

38 D. Moura, S. G. Caridade, M. P. Sousa, E. Cunha, H. Rocha, J. F. Mano, M. C. Paiva and N. M. Alves, J. Mater. Chem. B, 2016, 2(47), 7718-7730.

$39 \mathrm{H}$. Rocha, MSc thesis, University of Minho, 2014.

40 K. A. Mark, Biomacromolecules, 2003, 4(5), 1099-1120.

41 N. M. Alves, C. Picart and J. F. Mano, Macromol. Biosci., 2009, 9, 776-785.

42 M. V. Voinova, M. Rodahl, M. Jonson and B. Kasemo, Phys. Scr., 1999, 59(5), 391.

43 M. E. Gomes, H. S. Azevedo, A. R. Moreira, V. Ellä, M. Kellomäki and R. L. Reis, J. Tissue Eng. Regener. Med., 2008, 2, 243-252.

44 S. Park, C. T. Hung and G. A. Ateshian, Osteoarthritis Cartilage, 2004, 12(1), 65-73.

45 Z. Ni, Y. Wang, T. Yu and Z. Shen, Nano Res., 2008, 1(4), 273291.

46 L. Bokobza and J. Zhang, eXPRESS Polym. Lett., 2012, 6(7), 601-608.
47 Y. Zhu, S. Murali, C. Wai, X. Li, J. W. Suk, J. R. Potts and R. S. Ruoff, Adv. Mater., 2010, 22(35), 3906-3924.

48 V. Singh, D. Joung, L. Zhai, S. Das, S. I. Khondaker and S. Seal, Prog. Mater. Sci., 2011, 56(8), 1178-1271.

49 A. C. Ferrari, J. C. Meyer, V. Scardaci, C. Casiraghi, M. Lazzeri, F. Mauri, S. Piscanec, D. Jiang, K. S. Novoselov, S. Roth and A. K. Geim, Phys. Rev. Lett., 2006, 97(18), 1-4.

50 Y. y. Wang, Z. h. Ni, T. Yu, Z. X. Shen, H. m. Wang, Y. h. Wu, W. Chen and A. T. S. Wee, J. Phys. Chem. C, 2008, 112(29), 10637-10640.

51 E. Cunha, M. F. Proença, F. Costa, A. J. Fernandes, M. A. C. Ferro, P. E. Lopes, M. González-Debs, M. MelleFranco, L. F. Deepak and M. C. Paiva, ChemistryOpen, 2015, 4, 115-119.

52 Y. Xu, Z. Liu, X. Zhang, Y. Wang, J. Tian, Y. Huang, Y. Ma, X. Zhang and Y. A. Chen, Adv. Mater., 2009, 21, 1275-1279.

53 W. W. Liu, J. N. Wang and X. X. Wang, Nanoscale, 2012, 4, 425-428.

54 L. Sun and B. Fugetsu, Mater. Lett., 2013, 109, 207-210.

55 W. Zou, Z. Du, Y. Liu, X. Yang, H. Li and C. z. Zhang, Compos. Sci. Technol., 2008, 68(15-16), 3259-3264.

56 S. M. Notley, M. Eriksson and L. Wågberg, J. Colloid Interface Sci., 2005, 292(1), 29-37.

57 S. G. Caridade, C. Monge, F. Gilde, T. Boudou, J. F. Mano and C. Picart, Biomacromolecules, 2013, 14, 1653-1660.

58 D. Han, L. Yan, W. Chen and W. Li, Carbohydr. Polym., 2011, 83, 653-658.

59 J. H. Li, N. Ren, J. Qiu, X. Mou and H. Liu, Int. J. Nanomed., 2013, 8, 3415-3426.

60 F. T. Zohora and A. Y. M. A. Azim, Eur. Sci. J., 2014, 10(21), 186-209.

61 C. S. Hajicharalambous, J. Lichter, W. T. Hix, M. Swierczewska, M. F. Rubner and P. Rajagopalan, Biomaterials, 2009, 30(23-24), 4029-4036.

62 M. Olek, J. Ostrander, S. Jurga, H. Möhwald, N. Kotov, K. Kempa and M. Giersig, Nano Lett., 2004, 4(10), 1889-1895.

63 M. M. Gudarzi and F. Sharif, eXPRESS Polym. Lett., 2012, 6, 1017-1031.

64 X. Wang, H. Bai, Z. Yao, A. Liu and G. Shi, J. Mater. Chem., 2010, 20, 9032-9036.

65 M. Ionita, M. A. Pandele and H. Iovu, Carbohydr. Polym., 2013, 94(1), 339-344.

66 J. Lee, H.-R. Chae, Y. J. Won, K. Lee, C. H. Lee, H. H. Lee, I. C. Kim and J. Lee, J. Membr. Sci., 2013, 448, 223-230.

67 R. L. Reis, N. M. Neves, J. F. Mano, M. E. Gomes, A. P. Marques and H. S. Azevedo, Natural-Based Polymers for Biomedical Applications, Woodhead Publishing, 2008.

68 M. E. Gomes and R. L. Reis, Int. Mater. Rev., 2004, 49(5), 261273.

69 H. S. Azevedo and R. L. Reis, Understanding the enzymatic degradation of biodegradable polymers and strategies to control their degradation rate, in Biodegradable Systems in Tissue Engineering and Regenerative Medicine, ed. R. L. Reis and J. San Roman, FL CRC Press, Boca Raton, 2005, vol. 12, pp. 177-201.

70 R. Justin and B. Chen, Carbohydr. Polym., 2014, 103(1), 7080. 
71 K. P. Menard, Dynamic Mechanical Analysis: A practical introduction, CRC Press, 2008.

72 J. M. Silva, A. R. Duarte, S. G. Caridade, C. Picart, R. L. Reis and J. F. Mano, Tailored freestanding multi layered membranes based on chitosan and alginate, Biomacromolecules, 2014, 15, 3817-3826.

73 S. G. Caridade, C. Monge, J. Almodóvar, R. Guillot, J. Lavaud, V. Josserand, J. L. Coll, J. F. Mano and C. Picart, Acta Biomater., 2015, 15, 139-149.

74 H. L. Fan, L. Wang, K. Zhao, N. Li, Z. Shi, Z. Ge and Z. Jin, Biomacromolecules, 2010, 11, 2345-2351.

75 L. Nie, C. Liu, J. Wang, Y. Shuai, X. Cui and L. Liu, Carbohydr. Polym., 2015, 117, 616-662.

76 S. G. Caridade, E. G. Merino, N. M. Alves, V. Z. Bermudez, A. R. Boccaccino and J. F. Mano, J. Mech. Behav. Biomed. Mater., 2013, 20, 173-183.

77 J. F. Mano, R. L. Reis and A. M. Cunha, Dynamic Mechanical Analysis in Polymers for Medical Applications, in Polymer Based Systems on Tissue Engineering: Replacement and Regeneration, ed. R. L. Reis and D. Cohn, Springer, Netherlands, Amsterdam, 2002, vol. 86, pp. 139-164.
78 X. Y. Qi, D. Yan, Z. Jiang, Y. K. Cao, Z. Z. Yu, F. Yavari and N. Koratkar, ACS Appl. Mater. Interfaces, 2011, 3(8), 31303133.

79 F. Wang, Y. Zhang, B. B. Zhang, R. Y. Hong, M. R. Kumar and C. R. Xie, Enhanced electrical conductivity and mechanical properties of ABS/EPDM composites filled with graphene, Composites, Part B, 2015, 83, 66-74.

80 T. N. Zhou, X. D. Qi and Q. Fu, eXPRESS Polym. Lett., 2013, 7, 747-755.

81 H.-B. Zhang, W. G. Zheng, Q. Yang, Y. Yang, J. W. Wang, Z. H. Lu, G. Y. Ji and Z.-Z. Yu, Polymer, 2010, 51, 1191-1196. 82 C. Silva, MSc thesis, University of Minho, 2016.

83 W. Li, X. Z. Tang, H. B. Zhang, Z. G. Jiang, Z. Z. Yiu, X. S. Du and Y. W. Mai, Carbon, 2011, 49(14), 4724-4730.

84 T. A. Horbett, J. J. Waldburger, B. D. Ratner and A. S. Hoffman, J. Biomed. Mater. Res., 1988, 22(5), 383-404.

85 S. M. Oliveira, N. M. Alves and J. F. Mano, J. Adhes. Sci. Technol., 2012, 1-21.

86 J. Kim, Y. R. Kim, Y. Kim, K. T. Lim, H. Seonwoo, S. Park, S.-P. Cho, B. H. Hong, P. H. Choung, T. D. Chung, Y. H. Choung and J. H. Chung, J. Mater. Chem. B, 2013, 1, 933. 